\title{
A Comprehensive In Silico Analysis of the Functional and Structural Impact of Nonsynonymous SNPs in the ABCA1 Transporter Gene
}

\author{
Francisco R. Marín-Martín, ${ }^{1}$ Cristina Soler-Rivas, ${ }^{1}$ \\ Roberto Martín-Hernández, ${ }^{2}$ and Arantxa Rodriguez-Casado ${ }^{2,3}$ \\ ${ }^{1}$ Department of Production and Characterization of New Foods, Institute of Food Science Research (CIAL), UAM-CSIC, \\ Campus de Cantoblanco, 28049 Madrid, Spain \\ ${ }^{2}$ IMDEA Food Institute, Campus de Cantoblanco, 28049 Madrid, Spain \\ ${ }^{3}$ Nutritional Genomics of the Cardiovascular Disease and Obesity, IMDEA Food Institute, Carretera Cantoblanco 8, \\ 28049 Madrid, Spain
}

Correspondence should be addressed to Arantxa Rodriguez-Casado; arantxa.rodriguez@imdea.org

Received 30 April 2014; Revised 7 July 2014; Accepted 24 July 2014; Published 19 August 2014

Academic Editor: Akihiro Inazu

Copyright (C) 2014 Francisco R. Marín-Martín et al. This is an open access article distributed under the Creative Commons Attribution License, which permits unrestricted use, distribution, and reproduction in any medium, provided the original work is properly cited.

\begin{abstract}
Disease phenotypes and defects in function can be traced to nonsynonymous single nucleotide polymorphisms (nsSNPs), which are important indicators of action sites and effective potential therapeutic approaches. Identification of deleterious nsSNPs is crucial to characterize the genetic basis of diseases, assess individual susceptibility to disease, determinate molecular and therapeutic targets, and predict clinical phenotypes. In this study using PolyPhen 2 and MutPred in silico algorithms, we analyzed the genetic variations that can alter the expression and function of the ABCA1 gene that causes the allelic disorders familial hypoalphalipoproteinemia and Tangier disease. Predictions were validated with published results from in vitro, in vivo, and human studies. Out of a total of 233 nsSNPs, 80 (34.33\%) were found deleterious by both methods. Among these 80 deleterious nsSNPs found, 29 (12.44\%) rare variants resulted highly deleterious with a probability $>0.8$. We have observed that mostly variants with verified functional effect in experimental studies are correctly predicted as damage variants by MutPred and PolyPhen 2 tools. Still, the controversial results of experimental approaches correspond to nsSNPs predicted as neutral by both methods, or contradictory predictions are obtained for them. A total of seventeen nsSNPs were predicted as deleterious by PolyPhen2, which resulted neutral by MutPred. Otherwise, forty two nsSNPs were predicted as deleterious by MutPred, which resulted neutral by PolyPhen2.
\end{abstract}

\section{Introduction}

Nonsynonymous single nucleotide polymorphisms (nsSNPs) are single base changes in coding regions that cause an amino acid substitution in the correspondent proteins. These missense variants constitute the most identifiable group of SNPs represented by a small $(<1 \%)$ proportion [1]. The nsSNPs might alter structure, stability, and function of proteins and produce the least conservative substitutions with drastic phenotypic consequences [2-5]. Studies suggest that about $60 \%$ of Mendelian diseases are caused by amino acid exchanges [6]. Thousands of associations between Mendelian and complex diseases reveal a phenotypic code that links each complex disorder to a unique set of Mendelian loci [7]. Discriminating disease-associated from neutral variants would help to understand the genotype/phenotype relation and to develop diagnosis and treatment strategies for diseases. Nonetheless, the most important application is the evaluation of functional effect and impact of genomic variation, relating interactions with phenotypes translating the finding into medical practices.

ATP-binding cassette transporter $\mathrm{ABCA1}$ gene also known as the cholesterol efflux regulatory protein (CERP) encodes a $220 \mathrm{kDa}$ protein [8]. This protein is crucial for reverse 


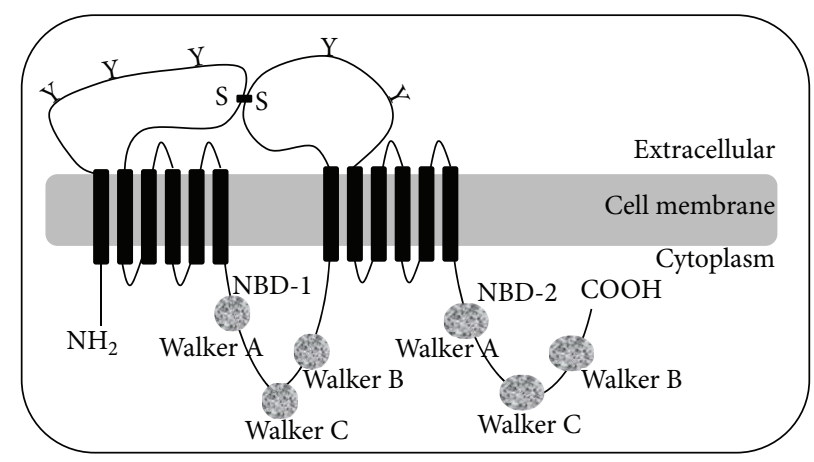

FIGURE 1: Predicted structure of ABCA1 [21, 23, 105]. The protein consists of 2261 amino acids and comprises 2 halves of similar structure. Each half encodes a transmembrane domain containing 6 helices (1-6 and 7-12) and 1 nucleotide binding domain (NBD-1 and NBD-2), where ATP is bound and utilized as energy for substrate transport across the membrane. Each NBD contains 2 highly conserved peptide motifs known as Walker $\mathrm{A}$ and Walker $\mathrm{B}$, which are present in many proteins that use $\mathrm{ATP}$ and a Walker $\mathrm{C}$ signature unique to $\mathrm{ABC}$ transporters. $\mathrm{ABCA1}$ is predicted to have an $\mathrm{N}$ terminus oriented into the cytosol and two large extracellular loops that are highly glycosylated and linked by cysteine bonds (Y indicates the glycosylation sites, and S-S indicates predicted disulfide bonds).

cholesterol transport and is considered as an important target in antiatherosclerosis treatment. ABCA1 mediates the efflux of cholesterol and phospholipids to lipid-poor apolipoproteins (apoAl and apoE), which form nascent high-density lipoproteins (HDL). ABCA1 resides on the cell membrane and has an extensive intracellular pathway, with rapid movement of the transporter between the cell membrane and intracellular vesicles [9]. ABCA1 is present in higher quantities in tissues that transfer or are involved in the turnover of lipids such as the liver, the small intestine, and adipose tissue [1012]. As well, lipid export activity of ABCA1 improves the function of pancreatic cells and ameliorates insulin release [13], reduces biliary cholesterol content protecting against gallstone [14], and plays a key role in lipid homeostasis in the lung [15]. Besides, evidence suggest a causal link between ABCA1 as cholesterol transporter and its antitumor activity $[16,17]$, as well as its implication in brain cholesterol homeostasis [15-20] founding lipid and myelin abnormalities in schizophrenia and Alzheimer's disease [18-20].

Although the entire ABCA1 protein 3D-structure remains unknown electron microscopic studies suggest a structural model consisting of a transmembrane domain (TMD) and a nucleotide-binding domain (NBD) (Figure 1) [14, 21, 22], where an NBD-TMD dimer is the minimum unit required for transport function $[22,23]$. Also, X-ray structures are available for different domains in the C-terminus protein essential for lipid efflux activity [24, 25]. Many variants disrupting the normal ABCA1 protein function result in modest or no circulating HDL [26-32]. Cholesterol accumulated within cells produces a toxicity that impairs cell function leading to a diversity of phenotypes, from severe disease states to mild impacts on health. In fact, the ABCA1 variability is associated with myocardial infarction, cancer, type 2 diabetes, and metabolic syndrome [33]. Heterozygous states, nearly one-third of them, are associated with hypoalphalipoproteinemia, known as familial HDL deficiency syndrome (FHA). Two copies cause a more severe syndrome Tangier disease (TD) [34-38] described by reduced HDL-c plasma level $(<5 \%)$, impaired cholesterol efflux, and a trend to accumulate intracellular cholesterol [34-43]. Indeed, loss of function of ABCA1 mutations in TD patients has a major impact on lipoprotein metabolism. A failure to acquire apolipoproteins leads to a rapid catabolism of lipid-poor apoA1 and accumulation of lipids in macrophages, intestinal cells, platelet, and hepatocytes [34$38,44]$. Compared with unaffected family members, heterozygotes and homozygotes have a more prevalent, premature, and severe atherosclerosis [42].

Because high levels of HDL-c are atheroprotective there is considerable interest in developing agents that act to increase ABCA1 expression and thereby raise plasma HDL-c levels. The nsSNPs are important indicators of action sites and effective potential therapeutic approaches. Therefore, it is crucial to identify deleterious nsSNPs to characterize the genetic basis of diseases, assess individual susceptibility to these diseases, determinate molecular and therapeutic targets, and predict clinical phenotypes. Beyond the genetic level, a disease depends on the sequence and the structural location of the nsSNPs of the protein. While the nsSNPs occur all through the ABCA1 gene, they tend to cluster in the extracellular loops, the NBD, and the COOH-terminal region (Figure 1). In fact, three structural motifs have been functionally associated with disease: the ARA motif, an interface between NBD and TMD that forms a partially buried $\alpha$-helix able to interact with the transmembrane helices, the conserved-loop 1, a allosteric loop between the membrane and globular domains, and the conserved-loop 2, an interaction surface for intracellular partners, critical in ATPbinding.

Even though many of nsSNP (rare or common) found in human ABCA1 have been identified, mainly in the HapMap project (http://hapmap.ncbi.nlm.nih.gov/), the molecular bases relating these variants and the caused phenotypes have not been studied in detail. To explore the effect of the large number of nsSNPs ABCA1 by experimental approaches would be extremely time-consuming and with low statistical chance. Alternatively, bioinformatic approaches, based on the biophysical severity of the amino acid exchange and the protein sequence and structural information, can offer a more 


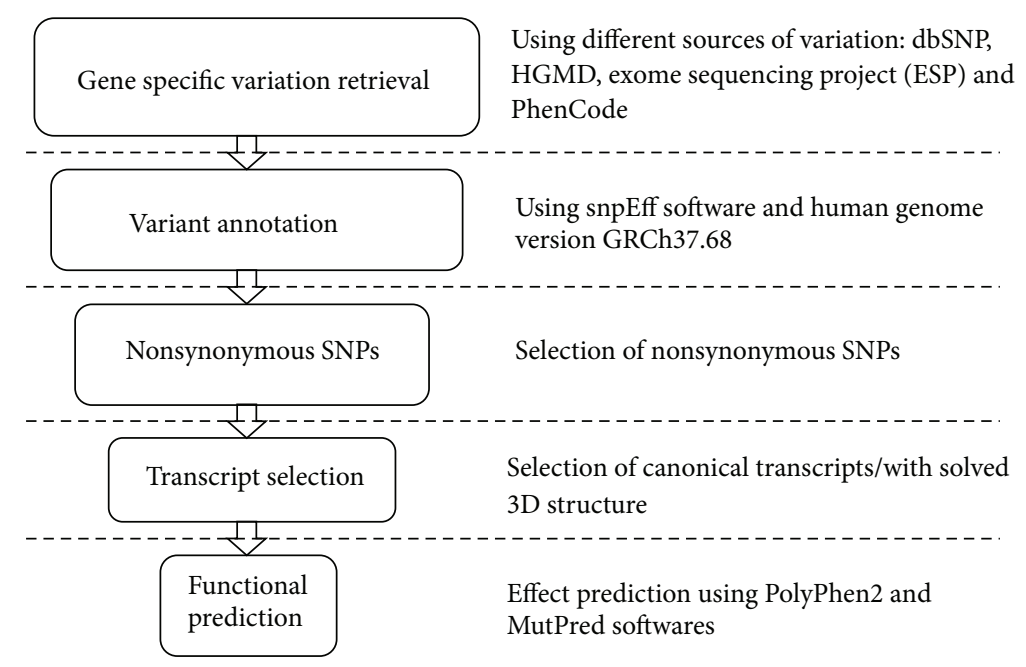

FIGURE 2: Workflow for predicting the effect of nsSNPs on ABCA1 protein. Gene specific variations were retrieved from the main variation databases. Mutations were annotated and nsSNPs belonging to the canonical transcript were selected in order to run the algorithms for functional prediction.

feasible phenotype prediction. As such, MutPred (mutation prediction) [45] and PolyPhen2 (polymorphism phenotyping 2) algorithms [46], were used in this study to investigate the impact of all known nsSNPs on ABCA1 protein function. Besides, based on the results of in vitro, in vivo, and human studies of this gene in the literature we validated the predictions made by reviewing the effect of the most critical nsSNPs in $\mathrm{ABCA1}$ gene and its pathological consequences.

\section{Methods}

Figure 2 shows the workflow designed to predict the nsSNPs effect on ABCA1 protein. ABCA1 human gene variants including SNPs, short insertions, and deletions were retrieved from Ensemble Variation 72 database 3141. Mutations were annotated using the SnpEff v3.2 toolbox [47] based on the human genome assembly GRCh37.68. Only variants found on the canonical transcript were considered for functional effect prediction, for what we used two different algorithms. PolyPhen2 (http://genetics.bwh.harvard.edu/pph2/) algorithm uses a naive Bayesian classifier to predict allele function based on a combination of sequence and structurebased attributes (if available) [46]. It calculates the probability for a given mutation to be benign, possibly damaging, or probably damaging. Then, we used MutPred (http://mutpred.mutdb.org/) [45] based upon SIFT algorithm [48] and a gain/loss of 14 predicted structural and functional properties. The predicted mutation outcome is based on a random forest (RF) classifier. The MutPred output includes the top 5 property scores and a general score (RF) equal to the probability of amino acid exchange is either deleterious or disease-associated. The ROC curves for both methods were generated using $\mathrm{R}$ programming language and the ROCR package (Figure 3) by a variation dataset obtained from VariBench (http://structure.bmc.lu.se/VariBench/) that contains mutations affecting protein tolerance including a neutral set of mutations comprising 17393 human coding nsSNPs and a pathogenic set of 14610 missense mutations obtained by manual curation from the PhenCode database.

Prediction accuracy accomplished by MutPred and PolyPhen2 depends on their specific criterion. Twelve structural and six sequence-based properties were used in this study (Table 1). About $28 \%$ of validated nsSNPs in the Human Genome Variation Database are predicted to affect protein function [49]. Similarly, about 25\% of nsSNPs affecting protein activity was predicted by PolyPhen2 [49]. MutPred offers classification accuracy with respect to human disease mutations. Considering conservative thresholds on the predicted disruption of molecular function, MutPred generates accurate and reliable hypotheses on the molecular basis of disease for about $11 \%$ of known inherited disease-causing mutations [45].

Our MutPred and PolyPhen2 predictions were validated by comparing them with previously obtained results from in vitro, in vivo, and human studies of ABCA1 gene in the databases and literature. When a given nsSNP found experimentally to be associated with a remarkable change of phenotype such as altered transporting activity or a disease was predicted by in silico methods as deleterious, it was considered that the prediction on this nsSNPs was correct. The prediction was defined as an error if such a deleterious nsSNP was predicted as tolerant.

\section{Results and Discussion}

The importance of ABCA1 in cholesterol efflux was demonstrated by the identification of ABCA1 mutations in TD and FHA families [34-38]. This has produced extensive research into the possibility to provide protection from atherosclerosis by increasing ABCA1 expression and thereby to raise plasma HDL-c levels. The identification of the large number of alleles for this transporter gene as target directly involved in HDL-c 
TABLE 1: Structural and functional properties used by MutPred and PolyPhen2.

\begin{tabular}{lll}
\hline & PolyPhen2 & \\
\hline Sequence based properties & & MutPred \\
Bond annotation & $\mathrm{X}$ & $\mathrm{X}$ \\
Functional site annotation & $\mathrm{X}$ & $\mathrm{X}$ \\
Region annotation & $\mathrm{X}$ & \\
PHAT score & $\mathrm{X}$ & \\
PSIC score & $\mathrm{X}$ & \\
SIFT score & & \\
Evolutionary attributes & $\mathrm{X}$ & \\
Structural properties & & \\
Secondary structure & $\mathrm{X}$ \\
Solvent-accessible surface area & $\mathrm{X}$ & \\
Phi-psi dihedral angles & $\mathrm{X}$ & \\
Normalized accessible surface area & $\mathrm{X}$ \\
Change in accessible surface propensity & $\mathrm{X}$ \\
Change in residue side chain volume & $\mathrm{X}$ \\
Region of the phi-psi map (Ramachandran map) & $\mathrm{X}$ \\
Normalized B-factor & $\mathrm{X}$ \\
Ligand contacts & $\mathrm{X}$ & $\mathrm{X}$ \\
Interchain contacts & $\mathrm{X}$ \\
Functional site contacts & $\mathrm{X}$ \\
Molecular Recognition Fragments (MoRFs) & \\
\hline
\end{tabular}

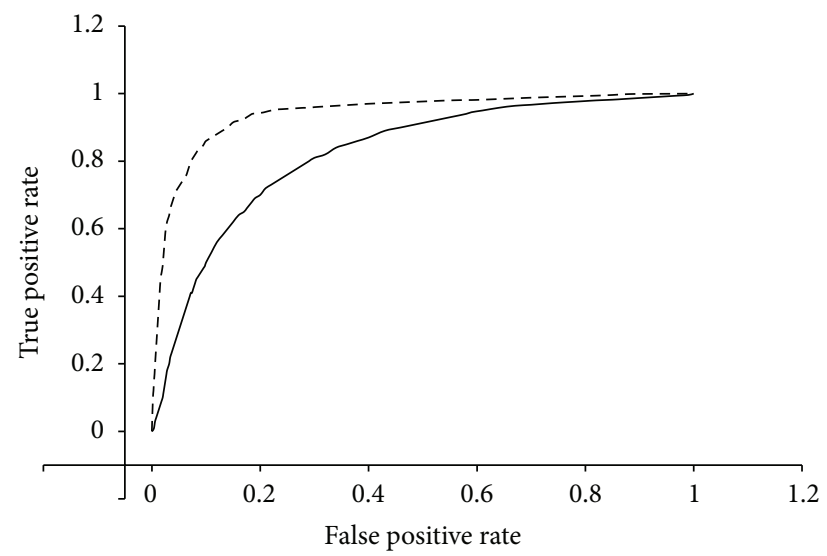

FIGURE 3: ROC (receiver operating characteristics) curve comparing the performance of MutPred and PolyPhen2 methods in predicting the outcome of nsSNPs. The dashed curve corresponds to MutPred predictions. As the area under the curve (AUC) for the MutPred method predictions is larger than the areas over the curve (AOC) which corresponds to the PolyPhen2 predictions curve, we can confirm that MutPred outperforms PolyPhen 2 in predicting the outcome of nsSNPs. The dataset used for the performance comparison was obtained from Varibench as stated in Methods section.

regulation constitutes a significant therapeutic strategy in reducing the risk for atherosclerosis.

3.1. Accuracy of the Prediction of the Functional Impact of $n s$ SNP. Out of a total of 3141 SNPs in ABCA1 gene retrieved from dbSNP, we found 233 nsSNPs, 126 sSNPs, 59 mRNA 3' UTR SNPs, 12 mRNA $5^{\prime}$-UTR SNPs, and 2543 intronic SNPs (Figure 4). Among the 233 nsSNPs, MutPred (RF score > $0.5)$ predicted $122(52.36 \%)$ as deleterious whereas PolyPhen2 (pph2_prob > 0.5) identified 97 (41.63\%) as potentially damaging and damaging. Then, once that MutPred was used to predict the nsSNP disease-association probability, the damaging probability of nsSNPs was validated by PolyPhen2. A total of $80(34.33 \%)$ nsSNPs were found to be deleterious by both methods. Among these 80 deleterious nsSNPs, the 29 (12.44\%) targeted (MAF/NA) that resulted with high pathological phenotype (probability > 0.8) are C1477R, W590L, W590S, A1046D, N1611D, M1091T, F2009S, N935H, R2081W, R1068H, N935S, R1068C, D1099Y, D1099N, W1699C, W840R, A937V, I1517R, C1660R, R1680W, P1065S, R1615P, T929I, Y2206D, L1379F, T940M, G1216V, Y2178H, and R1680Q. As shown in Table 2, a good correlation index was obtained 


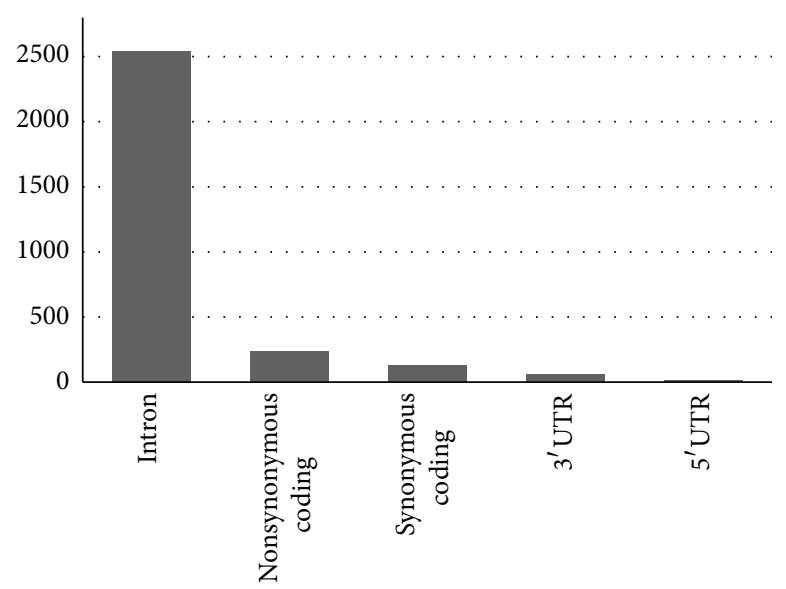

FIGURE 4: Distribution of ABCA1 nonsynonymous SNPs (nsSNPs), synonymous SNPs (sSNPs), $3^{\prime}$-UTR and $5^{\prime}$-UTR, and intronic SNPs.

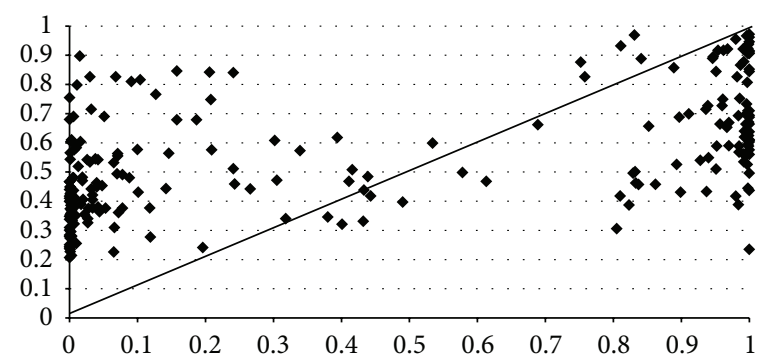

FIGURE 5: Scatter plot shows the correlation between the predictions made by PolyPhen2 ( $x$-axis) and MutPred ( $y$-axis) for 233 amino acid substitutions on ABCA1. The diagonal line represents a perfect correlation (=1) between both prediction methods for every mutation. The overall correlation of the predictions made by both methods is high (0.57). The majority of mutations classified as pathogenic by PolyPhen2 with the highest score $(=1)$ are also classified as pathogenic by MutPred but within a score range between 0.51 and 1 .

between the scores observed from the evolutionary-based approach MutPred and the structural-based approach PolyPhen2. As shown in Figure 5, the overall correlation of the predictions made by both methods is high $(\sim 0.57)$. The majority of mutations classified as pathogenic by PolyPhen 2 with the highest score $(=1)$ are also classified as pathogenic by MutPred but within a score range between 0.51 and 1 . The prediction accuracy depends not only by limitations of the in silico algorithms such as false positive error and interference of redundant motifs but also by the phenotype data from experimental studies [3].

Equally important is to consider the incorrect predictions in order to know the limitations of both algorithms and to suggest how they might be improved. Where MutPred predicts P2150L variant as deleterious, PolyPhen2 indicates a benign amino acid exchange. Conversely, MutPred predicted P85L to be probably damaging, while PolyPhen2 indicates it as neutral. Conflicting results were observed for a few other nsSNPs included in Table 2. A total of seventeen deleterious nsSNPs predicted by PolyPhen 2 resulted neutral by MutPred. In contrast, forty two deleterious nsSNPs by MutPred result neutral by PolyPhen2. We have observed (Figure 5, Table 2) that some mutational characteristics of nsSNPs such as C1477F, R666Q, P1475S, G616V, Q2210H, V1806M, and V304M show high PolyPhen2 values but very low MutPred scores due, at least in part, to loss or gain of catalytic residues and disorder and gain of ubiquitination and phosphorylation to the protein. On the other hand, some mutational characteristics of nsSNPs included T459P, A2028V, T774P, Q1279K, N1185K, D917N, E1005K, C887F, D1289N, Q188K, D462G, M1012I, R965C, S1181F, A255T, D457E, R496W, R1341T, R1925Q, R230C, L184S, R999L, and K1974R with low PolyPhen 2 values but high MutPred scores produce, however loss of solvent accessibility and of disorder, gain of phosphorylation, and both loss and gain of molecular recognition features (MoRFs) binding, loss and gain of methylation, and loss and gain of helix structure. Both, loss and gain of catalytic residues are actively involved in human inherited disease. Also, the small ubiquitin-a 76 residue $\beta$-grasp protein-is about $95 \%$ conserved from yeast to human. Overall, both gain and loss of a phosphorylation site in a target protein may be important features for predicting cancer-causing mutations and may represent a molecular cause of disease for a number of inherited and somatic mutations. Changes in secondary structure impair large functional alterations, as well as the solvent accessibility degree. Therefore, inaccurate predictions occurred at these sites could be explicated not only for the limited effects of genetic variant but also for geneenvironment interactions. Since the MutPred is based on a predicted structure of the protein under study rather than 


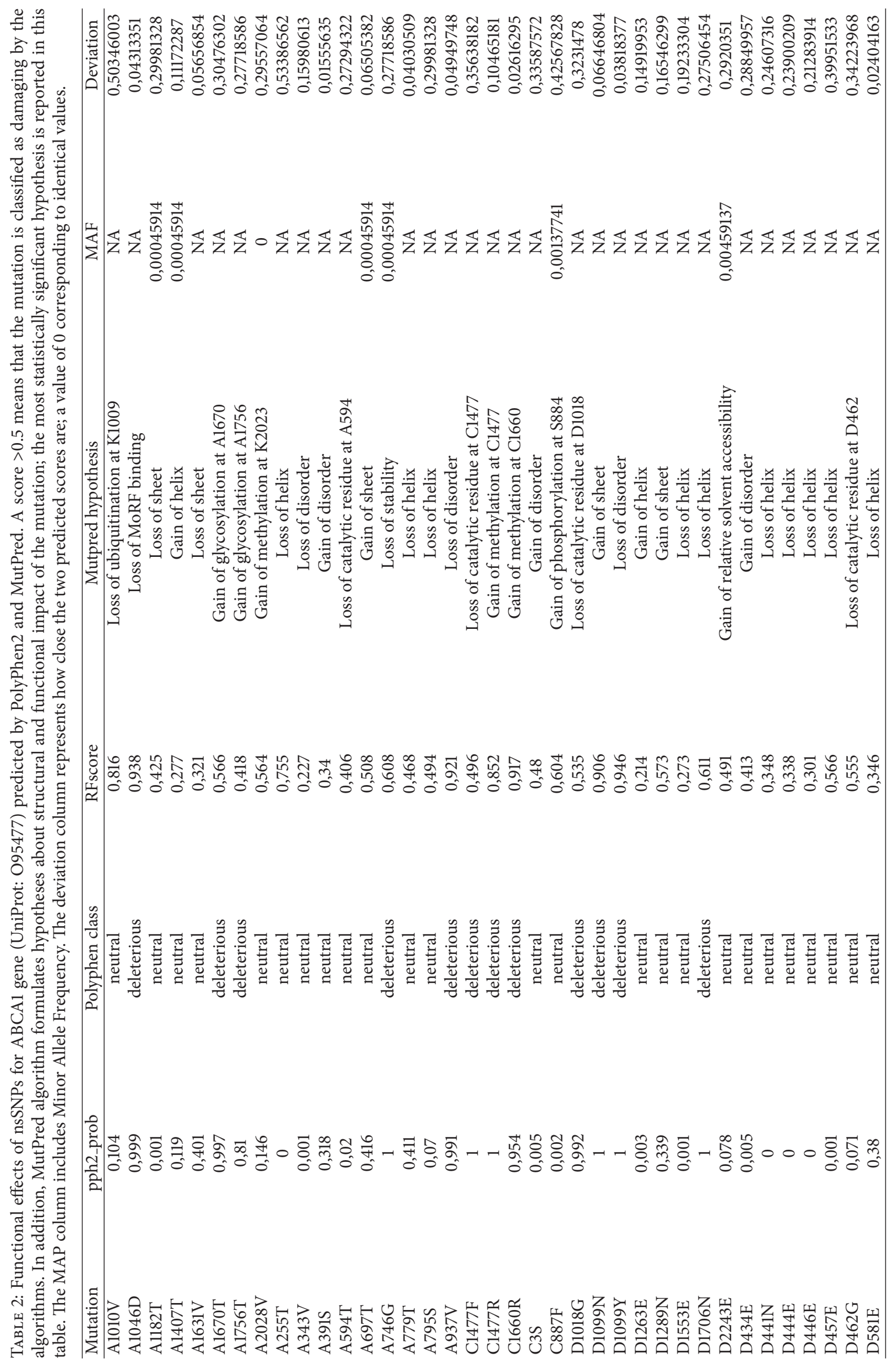




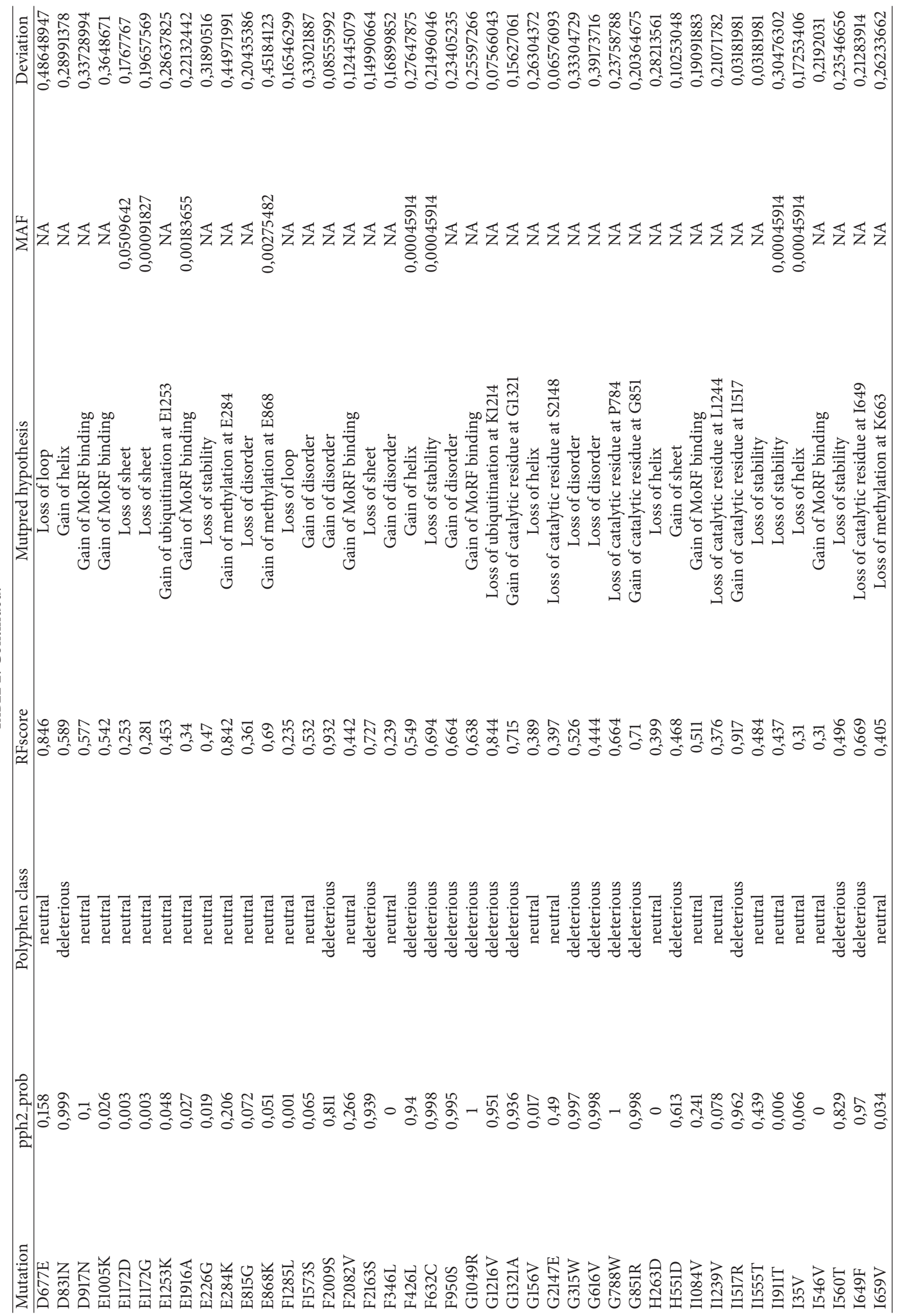




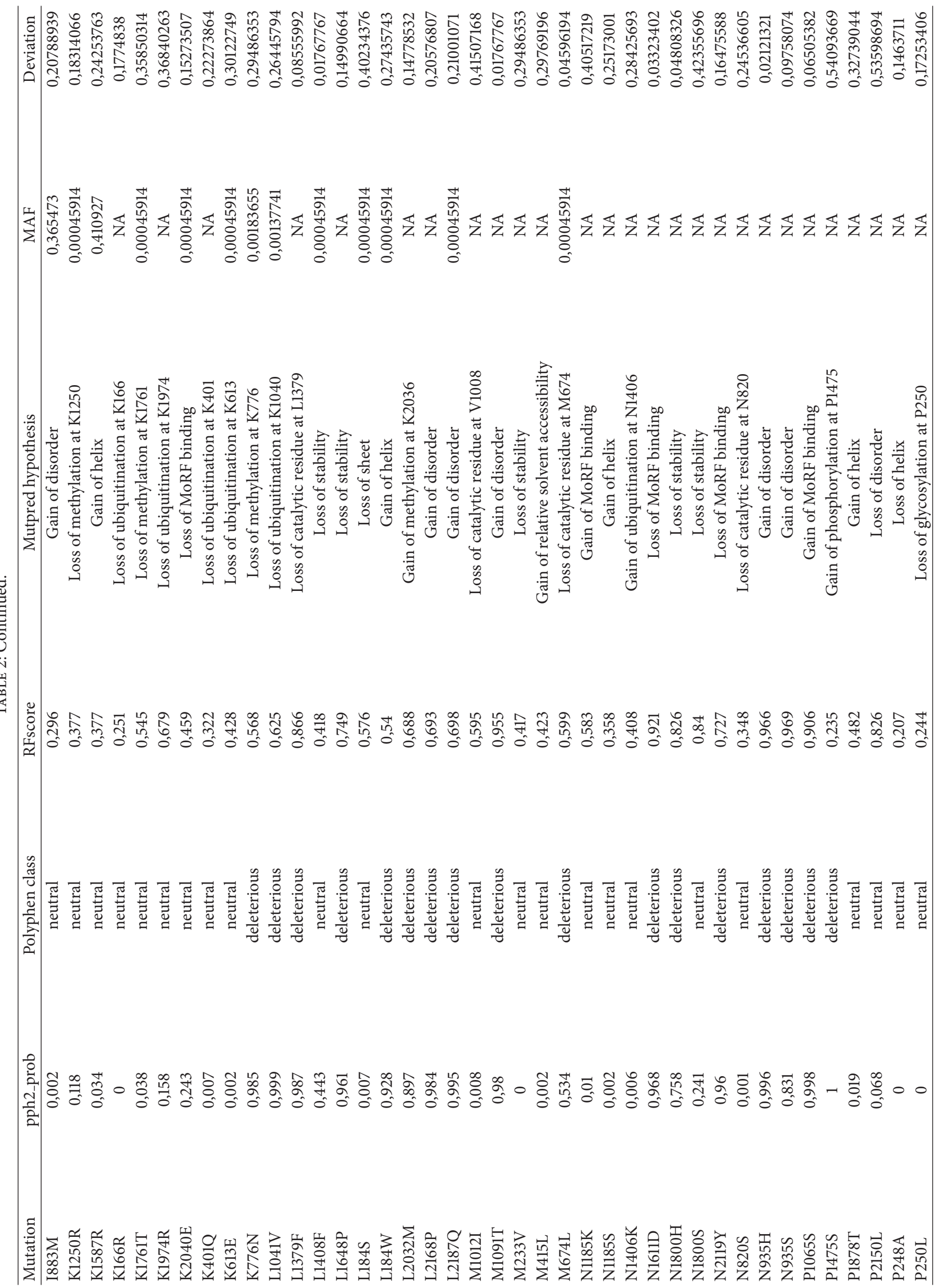




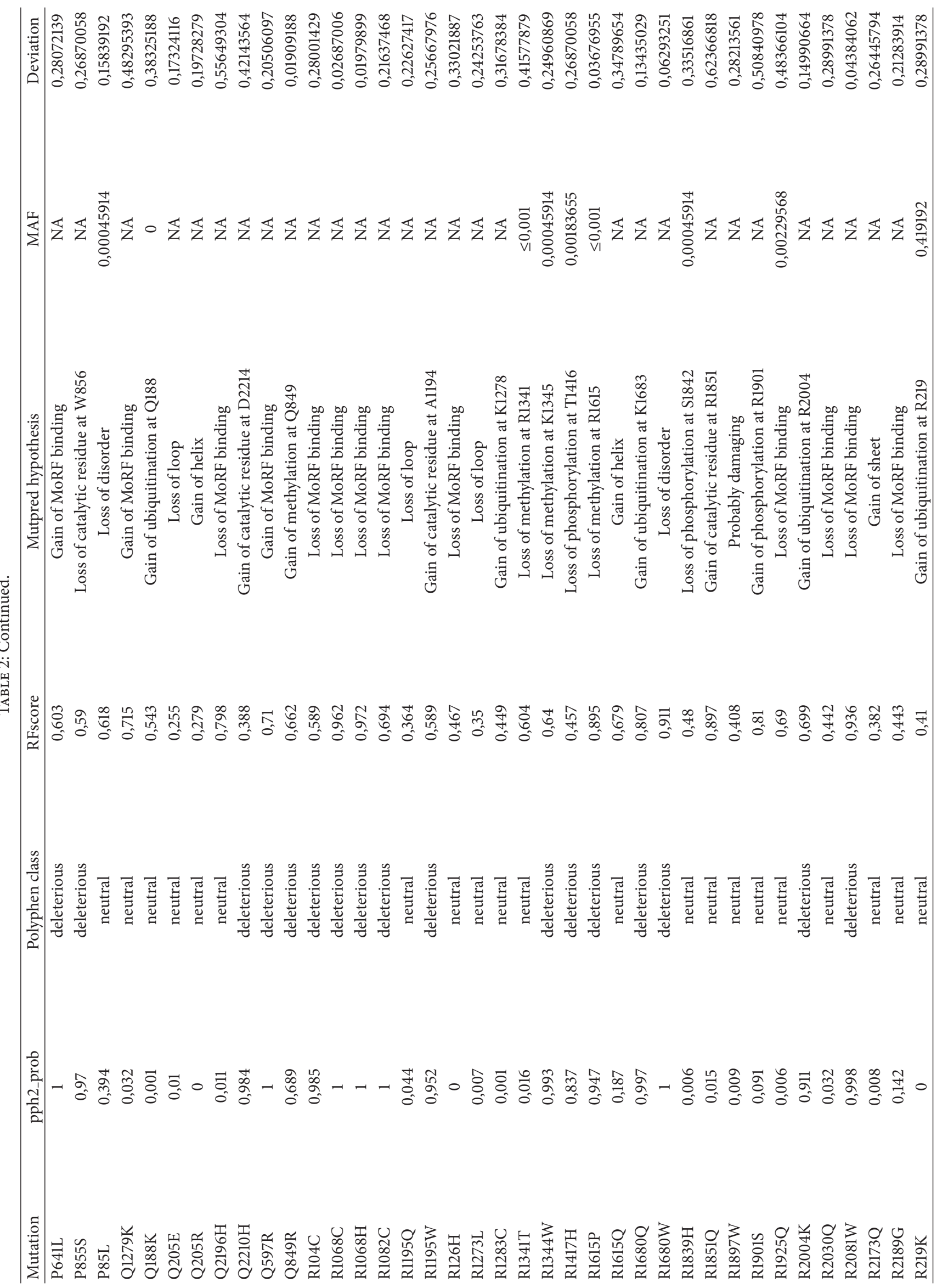




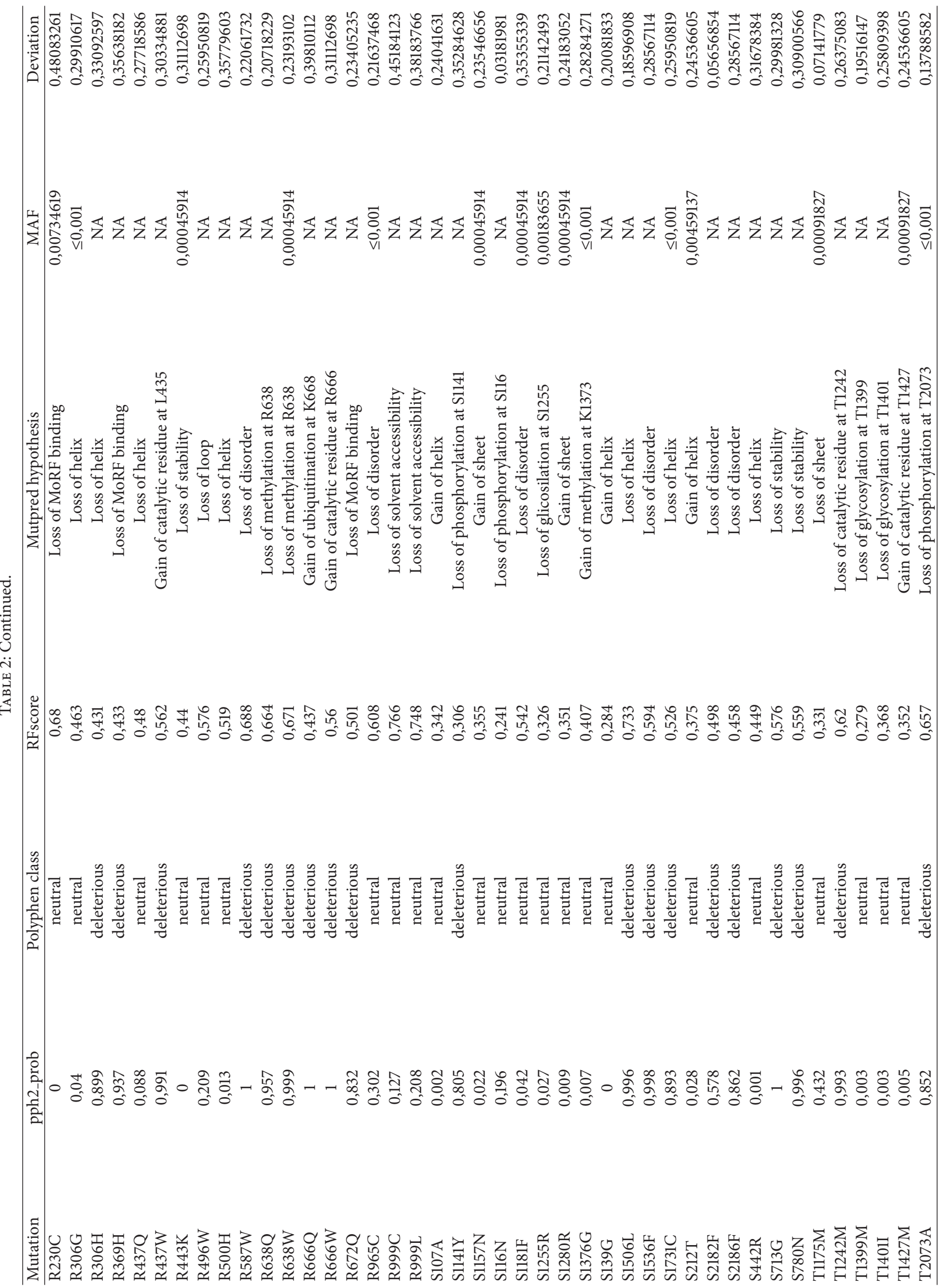




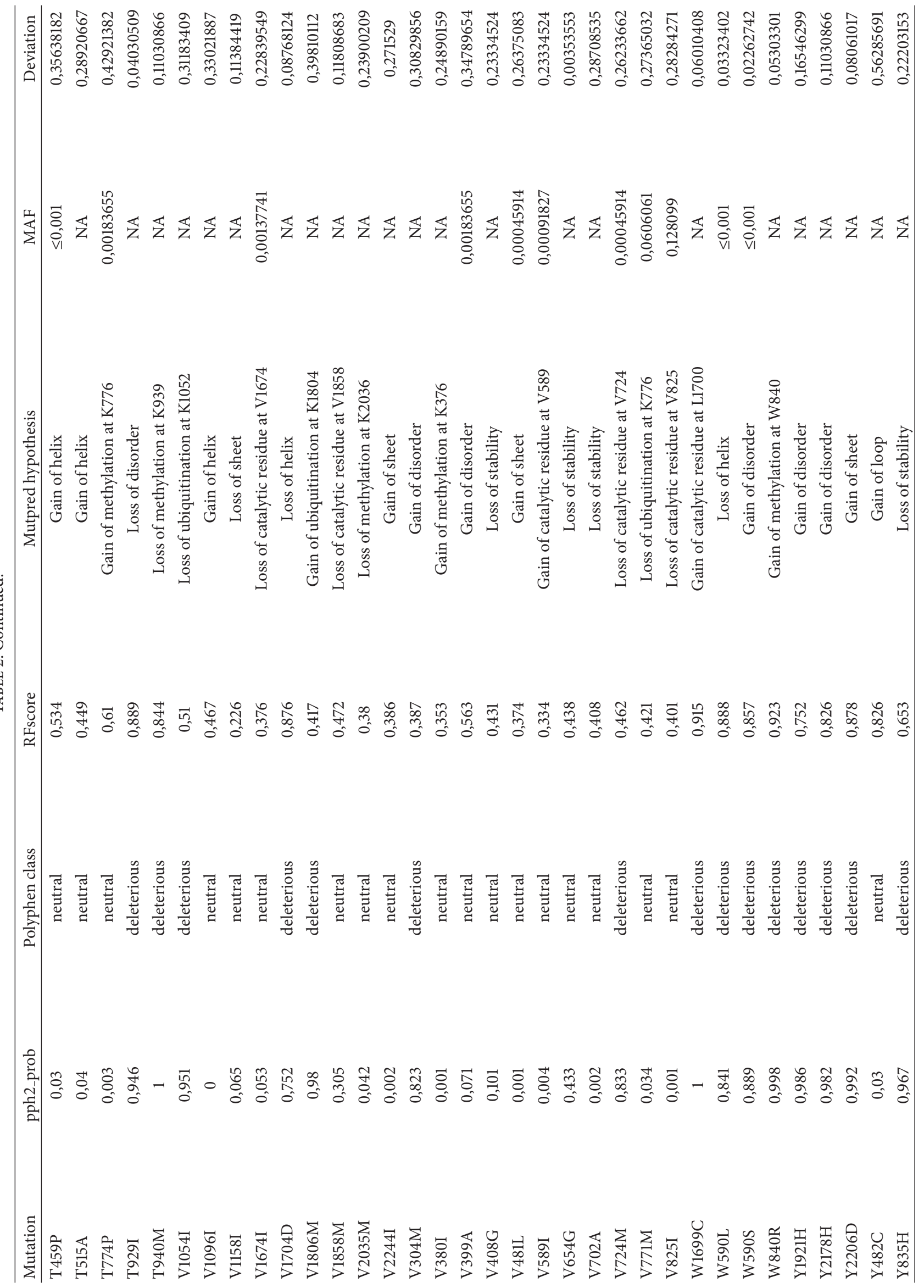


a solved structure as PolyPhen2 and considering the fact that nowadays the ABCA1 protein structure is only partially solved, it makes sense to prioritize the MutPred predictions. This fact was confirmed after evaluating the performance of both methods using a curated nsSNPs dataset with known outcome as a benchmark as shown in Figure 3. We have also observed that mostly, nsSNPs with verified functional effect in experimental studies are correctly predicted as damage variants by MutPred and PolyPhen 2 tools. Still, controversial experimental data are obtained for those nsSNPs predicted as neutral by both of these methods.

3.2. Functional Assessment of ABCA1 Variants. Disease-causing variants are under strong selective constraints, which determines if mutation frequency will increase, decrease, or change randomly during evolution. Most alterations are delseterious and so are finally removed during purifying selection. Benign mutations can sweep through the population and become fixed contributing to species differentiation. The ABCA1 gene is highly conserved between species. Human ABCA1 is $95.2 \%$ identical to mouse, $85.3 \%$ to chicken, $25.5 \%$ to drosophila, $21.6 \%$ to C. elegans, and $10.2 \%$ identical to fugu. In humans, there is an abundance of common nsSNPs that disrupt sites highly conserved across species and likely to be deleterious [50]. The information of nsSNPs can be used to outline the migration patterns of ancient humans and the ancestry of modern humans. Causal nsSNPs in single gene disorders are sufficient to impart large effects. Instead, complex traits are due to a much more complicated system of causative mechanisms that in aggregate increase the probability of disease. Genome-wide association studies reveal common genetic variants effects (common disease/common variant hypothesis) in complex traits. However, where common nsSNPs account for a relatively slight heritability of the traits, rare variants might produce large effects on the phenotype (rare variant/common disease hypothesis). The frequency range includes alleles that are exceptionally rare and even unique to an individual genome to be extremely common. Most deleterious nsSNPs are retained at lowpopulation frequencies due to negative selection. Thus, variants with large effect tend to be rare and those that exert weak effects are more common. It is worthy to note that rare alleles can also have weak effect or no effect. A specific locus may contain numerous rare alleles, so there may be many rare variants with large effect and a few common variants with weak effects. Although it has not yet been possible to determine whether other variables are associated with specific nsSNPs frequencies, variants within metabolic genes are not randomly distributed along the human population but follow diverse ethnic and/or geographic-specific patterns. It has been reported [51] that a significant proportion $(\sim 16 \%)$ of individuals with low HDL-c from the general population has the rare sequence of 25 variants in ABCA1 gene (Table 2, MAF $\leq 0.01$ ). However, consistent with MutPred and PolyPhen 2 only nine of them, N1800H, W590L, S1731C, C1477R, D1706N, R1615P, R638Q, T2073A, and A1670T, are predicted as functionally impaired. Some deleterious mutations from some other genes have reached intermediate to high frequencies. Specifically, the ancestral APOE4 allele, remains higher in populations like Pygmies (0.41), Khoi San (0.37), Papuans (0.37), some Native Americans (0.28), Lapps (0.31) and aborigines of Malaysia (0.24), and Australia (0.26) [52]. The exposure of APOE4 to the current environmental conditions could have rendered it a susceptibility allele for cardiovascular and Alzheimer diseases. However, the prediction for variant within ABCA1 gene indicates lack of harmful alleles to $\mathrm{MAF} \geq 0.01$. Therefore we have evaluated and contrasted the predictions made for nsSNPs (rare/common) most widely studied for their role in cholesterol pathway by reviewing the effect of the most significant nsSNPs in ABCA1 gene and its pathological consequences.

3.2.1. Accurate Prediction of the Functionally Deleterious nsSNPs in the ABCA1 Gene. The $\mathbf{N 1 8 0 0 H ~ A B C A 1 ~ h a s ~ b e e n ~ f u l l y ~}$ characterized showing a complete lack of protein function in terms of cholesterol efflux and HDL production $[53,54]$. Unlike the WT (wild-type), which is found at the endoplasmic reticulum and plasma membrane, $\mathrm{N} 1800 \mathrm{H}$ is accumulated intracellularly [54]. Even similar physicochemical properties (polar, medium size) of exchanged residues the $\mathrm{N} 1800 \mathrm{H}$ nsSNP, located between transmembrane domains [54], is a critical site for protein function. Scores from in silico methods predict the $\mathrm{N} 1800 \mathrm{H}$ variant as highly deleterious.

The W590L was never studied, but the W590S ABCA1 variant affecting the same position is extensively known [54]. Distribution of W590S is identical to WT [55] as well as apoA1 binding activity [54-57]; however it shows defective lipid transport $[54,56,58,59]$. Since multiple alignments often show a leucine residue in this position, it could be assumed that W590L had a similar behavior or even a lower impact than W590S on the protein function. Both W590S and W590L were predicted as deleterious nsSNPs with loss of functionality.

Studies indicate that S1731C variant alters the activity of ABCA1 protein $[27,51,60]$. This allele is present in FrenchCanadian families with low HDL-c levels [27] but not in subjects with normal [60] or high [51] HDL-c levels. Compared with WT, heterozygous show decreased $\sim 60 \%$ the cholesterol efflux activity $[27,51,61]$. Interestingly, some but not all families harboring S1731C also carried the 2144X stop mutation [60] able to produce the most severe effects on HDL-c levels and on cholesterol efflux [62]. These data along with our in silico predictions indicate that conserved S1731C is highly likely to affect protein function.

S1506L, Q597R, and C1477R variants are linked to TD and FHA and found in tumor cancer [54]. Normal function of ABCA1 inhibits tumor growth in human cancer cells [54]. However, although expressed to similar levels as WT, these alleles show deficient cellular cholesterol efflux and HDL production and do not decrease tumor growth [17]. The three are located intracellularly but C1477R is also found in membrane [54], which indicates that membrane localization is essential but not sufficient for apoA1 binding $[54,63]$. In fact, ApoA1 binds to ABCA1 protein oligomers but not with monomers [64]. Thus, conformation changes in binding sites might be produced by these nsSNPs found as deleterious by our in silico analysis. 
The $\mathbf{R 5 8 7} \mathrm{W}$ reaches the cell surface but reduces the apoA1 binding efficiency 50\% [56]. Others studies indicate that this allele is mainly retained intracellularly decreasing cholesterol efflux and apoA1 binding 75\% [54]. Severe HDL deficiency [34] and premature CVD is caused by R587W [65]. This variant is highly conserved during evolution, and the in silico analysis predicts it as strongly damaging and diseaseassociated. Besides to be related with TD, the R587W as well as W590S variants are linked to $\mathrm{AD}$. As the WT, these mutants significantly reduce $\mathrm{A} \beta$-peptide synthesis $\sim 45 \%$ [66], but increase by $\sim 2$-fold (R587W) and by $25 \%$ (W590S) amyloid precursor protein intracellular domain, a major cytotoxic of $\mathrm{AD}[66]$.

The A1046D, localized between conserved motifs [54, 67], shows an intermediate phenotype caused by its limited presence in the plasma membrane. This variant shows reduced apoAl binding efficacy, poor HDL-c, and folding protein alteration. Both in silico methods predict A1046D as a functional residue with a probability to be deleterious very close to 1 .

According to literature, N1611D is associated to probable atherosclerosis $[62,68]$. The mutated protein expression was comparable to WT although cholesterol efflux from the cells was markedly reduced. Our theoretical results indicate very high probabilities of this nsSNP being deleterious, which indicate an adverse and potential harmful effect on ABCA1 function.

The M1091T variant exerts a dominant-negative impact on ABCAl function with severe phenotype observed in subjects carrying this variant $[42,54,62]$. It is retained intracellularly preventing the protein from reaching the membrane [54]. In heterozygous, M1091T is lowered by 50\% HDL and inhibits apoAl binding and cholesterol efflux [54]. From evolutionary path, the inherited residue at this position has been methionine. Among related homologues ABCA2 and $\mathrm{ABCA} 4$ share a methionine at this position, while $\mathrm{ABCA7}$ substitutes a leucine. Consistent with this fact, $A B C A 1$ and $\mathrm{ABCA7}$ are functionally divergent, with $\mathrm{ABCA7}$ easing the efflux of phospholipids but not cholesterol [69]. Despite the modest conservation at this position, located in a critical cluster at the C-terminal region, in silico data suggest a severenegative impact.

The F2009S is conserved between human and mouse, that along with the exchange from large size and aromatic $(\mathrm{F})$ to small size and polar (S) explicates its reduced cholesterol efflux, low HDL-c, and apoAl levels [70]. The functional effect produced by F2009S variant is consistent with our prediction made by PolyPhen 2 and MutPred indicating a deleterious mutant.

The N935S variant is found intracellularly [54] in subjects without risk of premature atherosclerosis but with extremely low levels of HDL and signs of severe dementia and amyloid depositions in the brain $[71,72]$. This variant was predicted as deleterious by the used methods.

R1068H mutation is located within the first ATP-binding domain. It is identified in TD homozygous [73]. Since the $\mathrm{R} 1068 \mathrm{H}$ mutation is likely to produce a dysfunctional protein, one would expect it to be associated with FHA in the heterozygous state [73]. Residue R1068 is located in an $\alpha$-helix of the Walker B motif in the NBD, vulnerable to interaction with D1092 and E1093 [74]. Homology modeling of the $\mathrm{ABCA} 1$ protein showed that the $\mathrm{R} 1068 \mathrm{H}$ mutation disrupts the conformation of NBD. Functional studies of R1068H showed a lack of cholesterol efflux activity due to defective transference to the plasma membrane, mainly caused by impaired oligomerization [74]. The in silico analysis predicts a high possibility for $\mathrm{R} 1068 \mathrm{H}$ to be damaging. Besides, a different mutation of this position, $\mathbf{R} \mathbf{1 0 6 8 C}$, predicted as a deleterious by our methods, has been reported in a compound heterozygote with almost no HDL [31].

D1099Y is located at possible interaction site and exchanges the medium size and acidic residue to the large and aromatic tyrosine. Surface residues not at defined interfaces are usually preserved. Still, a moderate to highly conserved domain on the surface of the structure includes this nsSNP, which is associated to familial HDL deficiency $[70,75]$ and predicted as deleterious in our analysis.

The W1699C, located within the transmembrane domain, is accumulated within the cytoplasm and a small proportion reaches the plasma membrane [76]. It introduces a cysteine residue, which stimulates the formation of a new disulphide bridge able to disrupt the ABCA1 protein structure preventing its oligomerization and transference to the plasma membrane. Probably, W1699C retains some residual functions, as shown by the plasma HDL-c levels found in members carrying this mutation which were not as low as might be expected in carriers [76]. In silico analysis with PolyPhen2 and MutPred indicate a deleterious effect of this nsSNP on ABCA1 function.

\subsubsection{Controversial Results for Prediction of the Neutral nsS-} $N P$ s in the ABCAl Gene. The mutant R1897W that induces a change from basic (R) to aromatic (W) is predicted functionally neutral in this analysis. This variant was identified in the mother and the brother of an FHA patient, who had plasma HDL levels in the lower range of the normal values [77].

Both the D1289N and P2150L variants identified in TD patients are considered as disease causative $[42,78,79]$. Further experimental evidences disagreeing with these results suggest that both could be nonfunctional variants $[51,54,80]$. Indeed, they showed a lipid transport activity, apoA1 binding, and distribution similar to WT $[54,80]$. Interestingly, P2150L is only found in patients who also harbor a second variant, the deleterious R587W described above [54]. Besides, TD patients with D1289N variant were homozygous for a second mutation R2081W that could cause the shown pathological phenotype [79]. R2081W is missed at the plasma membrane and instead accumulated intracellularly [54]. Our results suggest that mutations R2081W and R587W are highly deleterious. For D1289N and P2150L variants, PolyPhen2 predicts a neutral impact on protein function contrary to MutPred predictions that indicates a high probability for these mutants to be deleterious. The positions 1289 and 2150 are conserved among all $\mathrm{ABCA1}$ orthologs but with the close-related ABCA7 and ABCA4. Since conservation patterns in ABCA1 protein endure for a relatively short time in evolutionary path, it is hard to determine if the conservation at these positions 
is due to functional constraint or simply reflects random chance. Along with experimental data, this suggests that $\mathrm{R} 2081 \mathrm{~W}$ is a major responsible of $\mathrm{ABCA} 1$ protein dysfunction found in TD patients.

The rare $\mathbf{2} 219 \mathrm{~K}$ polymorphism is located on an Nterminal extracellular loop, which mediates $\mathrm{ABCA1}$ protein interaction with apoA1 $[39,56,58,59]$. Despite high number of case-control studies conducted to investigate the functionality of R219K variant the results have been inconclusive $[60,81-83]$. While some reports suggest an association of $\mathrm{R} 219 \mathrm{~K}$ is with risk of CVD [84], other research indicates a decreased atherosclerosis progression in general population $[60,85]$. Conversely, large prospective studies found no association with HDL-c levels or atherosclerosis susceptibility $[82,86]$. A meta-analysis indicates that $\mathrm{R} 219 \mathrm{~K}$ polymorphism is protective against CVD in Asians but not in Caucasians [87]. Unexpectedly, the K219 allele was associated with a decreased risk of myocardial infarction $[18,60,84]$. Also, this variant has effect on triglycerides [60] but not with HDL-c [84] or with apoA1 levels [85]. Otherwise, a study indicates that blood lipid levels do not seem to be R219K dependent [88]. Whether this variant confers major susceptibility to CVD is for clarification. The association of R219K variant to risk of $\mathrm{AD}$ has been studied in diverse ethnic groups $[18-20,89,90]$. Although conflicting results were noted, a study observed a protective dependence in delaying the risk of late-onset $\mathrm{AD}$ [18]. Equal to other cases, experimental results inconclusive and contradictories result prediction of the $\mathrm{R} 219 \mathrm{~K}$ polymorphism was predicted to be neutral in our in silico analysis.

Some studies [60,91-93] but not all $[39,94]$ indicate that I883M variant severely increases the risk of atherosclerosis and $\mathrm{AD}$ [20]. The I883M has been reported as a milder phenotype with a significant reduction of HDL-c and cholesterol efflux ( $\sim 70 \%$ of WT) [51]. In contrast, others studies $[28,60,82,83,88]$ did not find any difference in lipid levels in I883M carriers. Studies among different healthy people $[95,96]$ as well as population with T2D [97] correlated the I883M variant with higher HDL-c concentration. Also, a stepwise regression approach identified I883M as one the key predictors of ischemic heart disease, whereas additive effects were found for V771M/I883M and I883M/E1172D pairs $[82,98]$. As well, several studies have reported associations between V825I/I883M and increased plasma HDL-c levels $[39,67,99]$. Despite the controversial experimental results on the influence on cholesterol efflux activity observed of this polymorphism, our data predict that the I883M variant is functionally neutral. Interestingly, both alleles are found in the human population and the minor allele, methionine, is likely to be the ancestral allele at this position. Along with the human ABCAlorthologs, murine aligns valine at this position and the chimpanzee sequence aligns methionine. This divergence could explain why a simple conservationbased approach predicts the I883M change as neutral.

The R1851Q variant exchanges the large size and basic arginine (R) residue to medium size and polar glutamine predicted deleterious by MutPred and neutral by PolyPhen 2 . R1851Q occurs within the extracellular loop proximal to the transmembrane $[68,100]$. Heterozygotes states show low
HDL-c and apoA1 levels compared with those related to WT protein.

The R230C variant, found in Native American groups but not in European, Asian, or African individuals, has been associated with low levels of HDL-c and apoA1 [101]. These results are confirmed after adjusting for gender, BMI, and waist circumference [102]. Besides, the C230 allele is associated with obesity, metabolic syndrome, and T2D in Mexican population [101]. Still, R230C may have conferred resistance against certain infectious diseases [101]. R230C has been reported as a rare variant causing FHA in an OjiCree individual [67]. MutPred predicts a high probability of functional impairment of R230C, while that the PolyPhen2 program predicts the variant as neutral. Other facts that suggest functionally damage are (1) R230C occurs at the first extracellular loop, where TD and FHA mutations are clustered; (2) the arginine at position 230 is conserved between species; and (3) very different nature of residues involved; whereas arginine is basic and hydrophilic, the hydrophobic cysteine is vulnerable to disulfide bond.

The variants, R1901S that induces a change from large size and basic (R) to small size and polar (S); Q2196H that exchanges residues with similar physicochemical property (medium size, polar); and E284K that exchanges a medium size and acidic (E) to large size and basic (K), are predicted to be deleterious by MutPred and neutral by PolyPhen2. The R1901S and Q2196H variants occur within the C-terminal domain, close to the NBD, and $\mathrm{E} 284 \mathrm{~K}$ was located in the first extracellular loop, all of them associated to FHA [76]. The A594T, I659V, T1512M, and R2004K polymorphisms display different degrees of mislocalization to the plasma membrane and slight impacts on cholesterol efflux [103]. These nsSNPs were identified in low-HDL subjects [29]. The A594T, I659V, and T1512M were predicted to be functionally neutral and the R2004K mutation possibly damaging [29]. Finally, the novel mutation (P85L) in ABCA1 was identified in one family with low HDL but was not detected in over 400 chromosomes of healthy subjects [104]. Our in silico prediction indicated this variant as possible damaging by MutPred and neutral by PolyPhen2.

\section{Conclusion and Future Directions}

The practice of medicine, including health promotion and disease prevention, is primarily based on phenotype-based approaches. Most of them are proximal phenotypes achieved through biochemical markers. Finding genetic determinants of the phenotypes could not only clarify biological and functional consequence of variants but also might translate and extend to clinical phenotype. This focus would consider the large locus heterogeneity and numerous nongenetic factors to contribute to the phenotype. Since high levels of HDL-c are atheroprotective, there is extensive interest in developing agents that enhance ABCA1 expression and thereby raise plasma HDL-c levels. Amino acid exchange variants are crucial indicators of action sites and effective potential therapeutic approaches. In fact, nsSNPs represent disease modifiers capable of altering drug/nutrient response and potential targets vulnerable to environmental factors. 
Evaluation of 233 nsSNPs (rare or common) found in ABCA1 transporter indicates that the rare 29 (12.44\%) of them resulted to be highly deleterious with a probability $>0.8$. From 20 sequence variants found in about $16 \%$ of individuals with low HDL cholesterol only nine of them, D1706N, R1615P, W590L, C1477R, N1800H, R638Q, T2073A, A1670T, and S1731C, were predicted by MutPred and PolyPhen2 as functionally impaired. We have observed that mostly nsSNPs with verified functional effect in all experimental studies made are correctly predicted as damage variants by MutPred and PolyPhen 2 tools. However, controversial experimental data are obtained for those nsSNPs predicted as neutral by both methods. Presumably clinical phenotype is the result of the additive effects and interactions among multiple alleles with different effect degree. Multiple rare alleles in ABCA1 contribute to plasma HDL-c levels in the general population.

Predicting the phenotypic consequence of nsSNPs using computational algorithms provides a better understanding of genetic differences in susceptibility to diseases and drug/ nutrient response. These methods predict whether an amino acid altering mutation is deleterious or disease-causing based on physicochemical properties, population frequency, protein structure, and cross-species conservation. However, computational prediction tools are generally based on machine learning algorithms, which need to be trained before classifying a mutation as either neutral or deleterious. A major obstacle of these approaches is the lack of experimentally validated and impartial data sets. A further complication is that mutations in highly conserved sequences do not always produce phenotypes that are easily noticeable. Besides, knowledge of protein structure is crucial to accurately predict functional nsSNPs and understand their linkage with disease. Severe limitation arises thus when protein 3D-structure is not available as the ABCA1 case. Thus, an accurate, efficient, and generally applicable approach is needed to establish a genotype/phenotype correlation. Whole genome sequencing is likely to become a commodity that could be readily available at a reasonable cost and be easily accommodated into the decision making tree of health care of every individual. The challenging task will be to identify variants that are disease-causing or likely disease-causing and develop strategies to prevent and attenuate the evolving phenotype. Likewise, various complementary studies, genetic and biological, would be necessary to discern the associated alleles from the true disease-causing variants. Moreover a better understanding of genome components, such as functional, large intergenic noncoding RNAs, small noncoding RNAs, and primary transcripts, would be essential. An integrated approach that utilizes genomics, transcriptomic, proteomics, and metabolomic would be expected to facilitate identification and characterization of the mechanisms involved in the pathogenesis of the phenotype.

\section{Conflict of Interests}

The authors declare that there is no conflict of interests regarding the publishing of this paper.

\section{Acknowledgment}

This work has been supported by European Union Structural Funds.

\section{References}

[1] E. Pennisi, "ENCODE project writes eulogy for junk DNA," Science, vol. 337, no. 6099, pp. 1159-1161, 2012.

[2] C. M. Yates and M. J. E. Sternberg, "The effects of non-synonymous single nucleotide polymorphisms (nsSNPs) on proteinprotein interactions," Journal of Molecular Biology, vol. 425, no. 21, pp. 3949-3969, 2013.

[3] P. C. Ng and S. Henikoff, "Predicting the effects of amino acid substitutions on protein function," Annual Review of Genomics and Human Genetics, vol. 7, pp. 61-80, 2006.

[4] M. González-Castejón, F. Marín, C. Soler-Rivas, G. Reglero, F. Visioli, and A. Rodríguez-Casado, "Functional non-synonymous polymorphisms prediction methods: current approaches and future developments," Current Medicinal Chemistry, vol. 18, no. 33, pp. 5095-5103, 2011.

[5] A. Rodriguez-Casado, "In silico investigation of functional nsSNPs an approach to rational drug design," Research and Reports in Medicinal Chemistry, vol. 2, pp. 31-42, 2012.

[6] D. Botstein and N. Risch, "Discovering genotypes underlying human phenotypes: past successes for mendelian disease, future approaches for complex disease," Nature Genetics, vol. 33, pp. 228-237, 2003.

[7] D. R. Blair, C. S. Lyttle, and J. M. Mortensen, "A nondegenerate code of deleterious variants in Mendelian loci contributes to complex disease risk," Cell, vol. 155, no. 1, pp. 70-80, 2013.

[8] J. F. Oram, "HDL apolipoproteins and ABCA1 partners in the removal of excess cellular cholesterol," Arteriosclerosis, Thrombosis, and Vascular Biology, vol. 23, no. 5, pp. 720-727, 2003.

[9] S. Santamarina-Fojo, A. T. Remaley, E. B. Neufeld, and H.B. Brewer Jr., "Regulation and intracellular trafficking of the ABCA1 transporter," Journal of Lipid Research, vol. 42, no. 9, pp. $1339-1345,2001$.

[10] L. R. Brunham, J. K. Kruit, J. Iqbal et al., "Intestinal ABCA1 directly contributes to HDL biogenesis in vivo," Journal of Clinical Investigation, vol. 116, no. 4, pp. 1052-1062, 2006.

[11] J. M. Timmins, J. Lee, E. Boudyguina et al., "Targeted inactivation of hepatic Abcal causes profound hypoalphalipoproteinemia and kidney hypercatabolism of apoA-I," Journal of Clinical Investigation, vol. 115, no. 5, pp. 1333-1342, 2005.

[12] L. R. Brunham, R. R. Singaraja, M. Duong et al., "Tissue-specific roles of ABCA1 influence susceptibility to atherosclerosis," Arteriosclerosis, Thrombosis, and Vascular Biology, vol. 29, no. 4, pp. 548-554, 2009.

[13] L. R. Brunham, J. K. Kruit, T. D. Pape et al., “ $\beta$-cell ABCA1 influences insulin secretion, glucose homeostasis and response to thiazolidinedione treatment," Nature Medicine, vol. 13, no. 3, pp. 340-347, 2007.

[14] J. Lee, A. Shirk, J. F. Oram, S. P. Lee, and R. Kuver, "Polarized cholesterol and phospholipid efflux in cultured gall-bladder epithelial cells: evidence for an ABCA1-mediated pathway," Biochemical Journal, vol. 364, no. 2, pp. 475-484, 2002.

[15] J. McNeish, R. J. Aiello, D. Guyot et al., "High density lipoprotein deficiency and foam cell accumulation in mice with targeted disruption of ATp-binding cassette transporter-1," Proceedings of the National Academy of Sciences of the United States of America, vol. 97, no. 8, pp. 4245-4250, 2000. 
[16] T. Sjoblom, S. Jones, and L. D. Wood, "The consensus coding sequences of human breast and colorectal cancers," Science, vol. 314, no. 5797, pp. 268-274, 2006.

[17] B. Smith and H. Land, "Anticancer activity of the cholesterol exporter ABCA1 gene," Cell Reports, vol. 2, no. 3, pp. 580-590, 2012.

[18] M. A. Wollmer, J. R. Streffer, D. Lütjohann et al., "ABCA1 modulates CSF cholesterol levels and influences the age at onset of Alzheimer's disease," Neurobiology of Aging, vol. 24, no. 3, pp. 421-426, 2003.

[19] P. D. Sundar, E. Feingold, R. L. Minster, S. T. DeKosky, and M. I. Kamboh, "Gender-specific association of ATP-binding cassette transporter 1 (ABCA1) polymorphisms with the risk of lateonset Alzheimer's disease," Neurobiology of Aging, vol. 28, no. 6, pp. 856-862, 2007.

[20] M. Ota, T. Fujii, K. Nemoto et al., "A polymorphism of the ABCA1 gene confers susceptibility to schizophrenia and related brain changes," Progress in Neuro-Psychopharmacology and Biological Psychiatry, vol. 35, no. 8, pp. 1877-1883, 2011.

[21] M. F. Rosenberg, R. Callaghan, R. C. Ford, and C. F. Higgins, "Structure of the multidrug resistance p-glycoprotein to $2.5 \mathrm{~nm}$ resolution determined by electron microscopy and image analysis," The Journal of Biological Chemistry, vol. 272, no. 16, pp. 10685-10694, 1997.

[22] M. Dean, Y. Hamon, and G. Chimini, "The human ATPbinding cassette $(\mathrm{ABC})$ transporter superfamily," Journal of Lipid Research, vol. 42, no. 7, pp. 1007-1017, 2001.

[23] M. L. Fitzgerald, A. J. Mendez, K. J. Moore, L. P. Andersson, H. A. Panjeton, and M. W. Freeman, "ATP-binding cassette transporter A1 Contains an NH2-terminal signal anchor sequence that translocates the protein's first hydrophilic domain to the exoplasmic space," Journal of Biological Chemistry, vol. 276, no. 18, pp. 15137-15145, 2001.

[24] F. Scheffel, U. Demmer, E. Warkentin, A. Hülsmann, E. Schneider, and U. Ermler, "Structure of the ATPase subunit CysA of the putative sulfate ATP-binding cassette $(\mathrm{ABC})$ transporter from Alicyclobacillus acidocaldarius," FEBS Letters, vol. 579, no. 13, pp. 2953-2958, 2005.

[25] C. L. Reyes and G. Chang, "Structure of the ABC transporter MsbA in complex with ADP.vanadate and lipopolysaccharide," Science, vol. 308, no. 5724, pp. 1028-1031, 2005.

[26] R. S. Kiss, N. Kavaslar, K. Okuhira et al., "Genetic etiology of isolated low HDL syndrome: incidence and heterogeneity of efflux defects," Arteriosclerosis, Thrombosis, and Vascular Biology, vol. 27, no. 5, pp. 1139-1145, 2007.

[27] K. Alrasadi, I. L. Ruel, M. Marcil, and J. Genest, "Functional mutations of the ABCA1 gene in subjects of French-Canadian descent with HDL deficiency," Atherosclerosis, vol. 188, no. 2, pp. 281-291, 2006.

[28] M. Mantaring, J. Rhyne, S. Ho Hong, and M. Miller, "Genotypic variation in ATP-binding cassette transporter-1 (ABCA1) as contributors to the high and low high-density lipoproteincholesterol (HDL-C) phenotype," Translational Research, vol. 149, no. 4, pp. 205-210, 2007.

[29] T. L. Slatter, G. T. Jones, M. J. A. Williams, A. M. van Rij, and S. P. A. McCormick, "Novel rare mutations and promoter haplotypes in ABCA1 contribute to low-HDL-C levels," Clinical Genetics, vol. 73, no. 2, pp. 179-184, 2008.

[30] M. T. Chhabria, B. N. Suhagia, and S. B. Pathik, "HDL elevation and lipid lowering therapy: current scenario and future perspectives," Frontiers in Cardiovascular Drug Discovery, vol. 1, pp. 3260, 2010.
[31] M. C. Probst, H. Thumann, C. Aslanidis et al., "Screening for functional sequence variations and mutations in ABCA1," Atherosclerosis, vol. 175, no. 2, pp. 269-279, 2004.

[32] C. Albrecht, K. Baynes, A. Sardini et al., "Two novel missense mutations in ABCAl result in altered trafficking and cause severe autosomal recessive HDL deficiency," Biochimica et Biophysica Acta, vol. 1689, no. 1, pp. 47-57, 2004.

[33] M. Daimon, T. Kido, M. Baba et al., "Association of the ABCA1 gene polymorphisms with type 2 DM in a Japanese population," Biochemical and Biophysical Research Communications, vol. 329, no. 1, pp. 205-210, 2005.

[34] R. M. Lawn, D. P. Wade, M. R. Garvin et al., "The Tangier disease gene product $\mathrm{ABCl}$ controls the cellular apolipoproteinmediated lipid removal pathway," Journal of Clinical Investigation, vol. 104, no. 8, pp. R25-R31, 1999.

[35] A. von Eckardstein, C. Langer, T. Engel et al., "ATP binding cassette transporter ABCA1 modulates the secretion of apolipoprotein E from human monocyte-derived macrophages," The FASEB Journal, vol. 15, no. 9, pp. 1555-1561, 2001.

[36] M. R. Hayden, S. M. Clee, A. Brooks-Wilson, J. Genest Jr., A. Attie, and J. J. P. Kastelein, "Cholesterol efflux regulatory protein, Tangier disease and familial high density lipoprotein deficiency," Current Opinion in Lipidology, vol. 11, no. 2, pp. 117-122, 2000.

[37] M. Marcil, A. Brooks-Wilson, S. M. Clee et al., "Mutations in the $\mathrm{ABCl}$ gene in familial HDL deficiency with defective cholesterol efflux," The Lancet, vol. 354, no. 9187, pp. 1341-1346, 1999.

[38] H. H. Hobbs and D. J. Rader, "ABC1: connecting yellow tonsils, neuropathy, and very low HDL," The Journal of Clinical Investigation, vol. 104, no. 8, pp. 1015-1017, 1999.

[39] R. Frikke-Schmidt, B. G. Nordestgaard, G. B. Jensen, and A. Tybjærg-Hansen, "Genetic variation in ABC transporter A1 contributes to HDL cholesterol in the general population," The Journal of Clinical Investigation, vol. 114, no. 9, pp. 1343-1353, 2004.

[40] M. Marcil, L. Yu, L. Krimbou et al., "Cellular cholesterol transport and efflux in fibroblasts are abnormal in subjects with familial HDL deficiency," Arteriosclerosis, Thrombosis, and Vascular Biology, vol. 19, no. 1, pp. 159-169, 1999.

[41] M. E. Brousseau, G. P. Eberhart, J. Dupuis et al., "Cellular cholesterol efflux in heterozygotes for Tangier disease is markedly reduced and correlates with high density lipoprotein cholesterol concentration and particle size," Journal of Lipid Research, vol. 41, no. 7, pp. 1125-1135, 2000.

[42] S. M. Clee, J. J. P. Kastelein, M. van Dam et al., "Age and residual cholesterol efflux affect HDL cholesterol levels and coronary artery disease in ABCA1 heterozygotes," The Journal of Clinical Investigation, vol. 106, no. 10, pp. 1263-1270, 2000.

[43] N. F. Fitz, A. A. Cronican, M. Saleem et al., "Abcal deficiency affects Alzheimer's disease-like phenotype in human ApoE4 but not in ApoE3-targeted replacement mice," Journal of Neuroscience, vol. 32, no. 38, pp. 13125-13136, 2012.

[44] E. J. Schaefer, L. A. Zech, D. E. Schwartz, and H. B. Brewer Jr., "Coronary heart disease prevalence and other clinical features in familial high-density lipoprotein deficiency (Tangier disease)," Annals of Internal Medicine, vol. 93, no. 2, pp. 261-266, 1980.

[45] B. Li, V. G. Krishnan, M. E. Mort et al., "Automated inference of molecular mechanisms of disease from amino acid substitutions," Bioinformatics, vol. 25, no. 21, pp. 2744-2750, 2009. 
[46] I. A. Adzhubei, S. Schmidt, L. Peshkin et al., "A method and server for predicting damaging missense mutations," Nature Methods, vol. 7, no. 4, pp. 248-249, 2010.

[47] P. Cingolani, A. Platts, L. L. Wang et al., "A program for annotating and predicting the effects of single nucleotide polymorphisms, SnpEff: SNPs in the genome of Drosophila melanogaster strain $\mathrm{w}^{1118}$; iso-2; iso-3," Fly, vol. 6, no. 2, pp. 80-92, 2012.

[48] P. Kumar, S. Henikoff, and P. C. Ng, "Predicting the effects of coding non-synonymous variants on protein function using the SIFT algorithm," Nature Protocols, vol. 4, no. 7, pp. 1073-1081, 2009.

[49] V. Ramensky, P. Bork, and S. Sunyaev, "Human non-synonymous SNPs: server and survey," Nucleic Acids Research, vol. 30, no. 17, pp. 3894-3900, 2002.

[50] S. Chun and J. C. Fay, "Identification of deleterious mutations within three human genomes," Genome Research, vol. 19, no. 9, pp. 1553-1561, 2009.

[51] J. C. Cohen, R. S. Kiss, A. Pertsemlidis, Y. L. Marcel, R. McPherson, and H. H. Hobbs, "Multiple rare alleles contribute to low plasma levels of HDL cholesterol," Science, vol. 305, no. 5685, pp. 869-872, 2004.

[52] R. M. Corbo and R. Scacchi, "Apolipoprotein E (APOE) allele distribution in the world. Is APOE* 4 a "thrifty" allele?" Annals of Human Genetics, vol. 63, part 4, pp. 301-310, 1999.

[53] D. Weissglas-Volkov and P. Pajukanta, "Genetic causes of high and low serum HDL-cholesterol," Journal of Lipid Research, vol. 51, no. 8, pp. 2032-2057, 2010.

[54] R. R. Singaraja, H. Visscher, E. R. James et al., "Specific mutations in ABCA1 have discrete effects on ABCA1 function and lipid phenotypes both in vivo and in vitro," Circulation Research, vol. 99, no. 4, pp. 389-397, 2006.

[55] K. Nagao, M. Tomioka, and K. Ueda, "Function and regulation of ABCA1-membrane meso-domain organization and reorganization," The FEBS Journal, vol. 278, no. 18, pp. 3190-3203, 2011.

[56] M. L. Fitzgerald, A. L. Morris, J. S. Rhee, L. P. Andersson, A. J. Mendez, and M. W. Freeman, "Naturally occurring mutations in the largest extracellular loops of ABCA1 can disrupt its direct interaction with apolipoprotein A-I," The Journal of Biological Chemistry, vol. 277, no. 36, pp. 33178-33187, 2002.

[57] A. M. Vaughan, C. Tang, and J. F. Oram, "ABCA1 mutants reveal an interdependency between lipid export function, apoA-I binding activity, and Janus kinase 2 activation," Journal of Lipid Research, vol. 50, no. 2, pp. 285-292, 2009.

[58] R. Frikke-Schmidt, C. F. Sing, B. G. Nordestgaard, and A. Tybjærg-Hansen, "Gender- and age-specific contributions of additional DNA sequence variation in the $5^{\prime}$ regulatory region of the APOE gene to prediction of measures of lipid metabolism," Human Genetics, vol. 115, no. 4, pp. 331-345, 2004.

[59] R. Frikke-Schmidt, B. G. Nordestgaard, P. Schnohr, and A. Tybjærg-Hansen, "Single nucleotide polymorphism in the lowdensity lipoprotein receptor is associated with a threefold risk of stroke: a case-control and prospective study," European Heart Journal, vol. 25, no. 11, pp. 943-951, 2004.

[60] S. M. Clee, A. H. Zwinderman, J. C. Engert et al., "Common genetic variation in ABCA1 is associated with altered lipoprotein levels and a modified risk for coronary artery disease," Circulation, vol. 103, no. 9, pp. 1198-1205, 2001.

[61] M. V. Reddy, I. Iatan, D. Weissglas-Volkov et al., "Exome sequencing identifies 2 rare variants for low high-density lipoprotein cholesterol in an extended family," Circulation: Cardiovascular Genetics, vol. 5, no. 5, pp. 538-546, 2012.
[62] C. L. Wellington, Y. Yang, S. Zhou et al., “Truncation mutations in ABCA1 suppress normal upregulation of full-length ABCA1 by 9-cis-retinoic acid and 22-R-hydroxycholesterol," Journal of Lipid Research, vol. 43, no. 11, pp. 1939-1949, 2002.

[63] S. Wang, K. Gulshan, G. Brubaker, S. L. Hazen, and J. D. Smith, "ABCA1 mediates unfolding of apolipoprotein AI N terminus on the cell surface before lipidation and release of nascent highdensity lipoprotein," Arteriosclerosis, Thrombosis, and Vascular Biology, vol. 33, no. 6, pp. 1197-1205, 2013.

[64] M. Denis, B. Haidar, M. Marcil, M. Bouvier, L. Krimbou, and J. Genest, "Characterization of oligomeric human ATP binding cassette transporter A1: potential implications for determining the structure of nascent high density lipoprotein particles," The Journal of Biological Chemistry, vol. 279, no. 40, pp. 4152941536, 2004.

[65] S. Bertolini, L. Pisciotta, M. Seri et al., "A point mutation in $\mathrm{ABCl}$ gene in a patient with severe premature coronary heart disease and mild clinical phenotype of Tangier disease," Atherosclerosis, vol. 154, no. 3, pp. 599-605, 2001.

[66] W. S. Kim, A. F. Hill, M. L. Fitzgerald, M. W. Freeman, G. Evin, and B. Garner, "Wild type and tangier disease ABCA1 mutants modulate cellular amyloid- $\beta$ production independent of cholesterol efflux activity," Journal of Alzheimer's Disease, vol. 27, no. 2, pp. 441-452, 2011.

[67] J. Wang, J. R. Burnett, S. Near et al., "Common and rare ABCA1 variants affecting plasma HDL cholesterol," Arteriosclerosis, Thrombosis, and Vascular Biology, vol. 20, no. 8, pp. 1983-1989, 2000.

[68] Y. Nishida, K. Hirano, K. Tsukamoto et al., "Expression and functional analyses of novel mutations of ATP-binding cassette transporter-1 in Japanese patients with high-density lipoprotein deficiency," Biochemical and Biophysical Research Communications, vol. 290, no. 2, pp. 713-721, 2002.

[69] N. Wang, D. Lan, M. Gerbod-Giannone et al., "ATP-binding cassette transporter A7 (ABCA7) binds apolipoprotein A-I and mediates cellular phospholipid but not cholesterol efflux," The Journal of Biological Chemistry, vol. 278, no. 44, pp. 4290642912, 2003.

[70] S. Ho Hong, J. Rhyne, K. Zeller, and M. Miller, "Novel ABCA1 compound variant associated with HDL cholesterol deficiency," Biochimica et Biophysica Acta-Molecular Basis of Disease, vol. 1587, no. 1, pp. 60-64, 2002.

[71] M. Walter, S. Kerber, C. Fechtrup, U. Seedorf, G. Breithardt, and G. Assmann, "Characterization of atherosclerosis in a patient with familial high-density lipoprotein deficiency," Atherosclerosis, vol. 110, no. 2, pp. 203-208, 1994.

[72] R. P. Koldamova, I. M. Lefterov, M. Staufenbiel et al., "The liver $\mathrm{X}$ receptor ligaun T0901317 decreases amyloid $\beta$ production in vitro and in a mouse model of Alzheimer's disease," The Journal of Biological Chemistry, vol. 280, no. 6, pp. 4079-4088, 2005.

[73] T. L. Slatter, M. J. A. Williams, R. Frikke-Schmidt, A. TybjærgHansen, I. M. Morison, and S. P. A. McCormick, "Promoter haplotype of a new ABCA1 mutant influences expression of familial hypoalphalipoproteinemia," Atherosclerosis, vol. 187, no. 2, pp. 393-400, 2006.

[74] R. J. Suetani, B. Sorrenson, J. D. A. Tyndall, M. J. A. Williams, and S. P. A. McCormick, "Homology modeling and functional testing of an ABCA1 mutation causing Tangier disease," Atherosclerosis, vol. 218, no. 2, pp. 404-410, 2011.

[75] L. Kelly, R. Karchin, and A. Sali, "Protein interactions and disease phenotypes in the $\mathrm{ABC}$ transporter superfamily," in 
Proceedings of the Pacific Symposium on Biocomputing, pp. 5163, 2007.

[76] L. Pisciotta, L. Bocchi, C. Candini et al., "Severe HDL deficiency due to novel defects in the ABCAl transporter," Journal of Internal Medicine, vol. 265, no. 3, pp. 359-372, 2009.

[77] T. Fasano, L. Bocchi, L. Pisciotta, S. Bertolini, and S. Calandra, "Denaturing high-performance liquid chromatography in the detection of ABCA1 gene mutations in familial HDL deficiency," Journal of Lipid Research, vol. 46, no. 4, pp. 817-822, 2005.

[78] M. E. Brousseau, E. J. Schaefer, J. Dupuis et al., "Novel mutations in the gene encoding ATP-binding cassette 1 in four tangier disease kindreds," Journal of Lipid Research, vol. 41, no. 3, pp. 433-441, 2000.

[79] W. Huang, K. Moriyama, T. Koga et al., "Novel mutations in ABCA1 gene in Japanese patients with Tangier disease and familial high density lipoprotein deficiency with coronary heart disease," Biochimica et Biophysica Acta, vol. 1537, no. 1, pp. 71-78, 2001.

[80] F. Quazi and R. S. Molday, "Differential phospholipid substrates and directional transport by ATP binding cassette proteins ABCA1, ABCA7, and ABCA4 and disease-causing mutants," The Journal of Biological Chemistry, vol. 288, no. 48, pp. 3441434426, 2013.

[81] V. Kolovou, G. Kolovou, A. Marvaki et al., "ATP-binding cassette transporter Al gene polymorphisms and serum lipid levels in young Greek nurses," Lipids in Health and Disease, vol. 10, article $56,2011$.

[82] R. Frikke-Schmidt, B. G. Nordestgaard, G. B. Jensen, R. Steffensen, and A. Tybjærg-Hansen, "Genetic variation in ABCA1 predicts ischemic heart disease in the general population," Arteriosclerosis, Thrombosis, and Vascular Biology, vol. 28, no. 1, pp. 180-186, 2008.

[83] A. Kitjaroentham, H. Hananantachai, A. Tungtrongchitr, S. Pooudong, and R. Tungtrongchitr, "R219K polymorphism of ATP binding cassette transporter A1 related with low HDL in overweight/obese Thai males," Archives of Medical Research, vol. 38, no. 8, pp. 834-838, 2007.

[84] A. Cenarro, M. Artieda, S. Castillo et al., "A common variant in the $A B C A 1$ gene is associated with a lower risk for premature coronary heart disease in familial hypercholesterolaemia," Journal of Medical Genetics, vol. 40, no. 3, pp. 163-168, 2003.

[85] D. Tregouet, S. Ricard, V. Nicaud et al., "In-depth haplotype analysis of ABCA1 gene polymorphisms in relation to plasma ApoA1 levels and myocardial infarction," Arteriosclerosis, Thrombosis, and Vascular Biology, vol. 24, no. 4, pp. 775-781, 2004.

[86] T. M. Morgan, H. M. Krumholz, R. P. Lifton, and J. A. Spertus, "Nonvalidation of reported genetic risk factors for acute coronary syndrome in a large-scale replication study," Journal of the American Medical Association, vol. 297, no. 14, pp. 1551$1561,2007$.

[87] Y. Li, K. Tang, K. Zhou et al., "Quantitative assessment of the effect of ABCA1 R219K polymorphism on the risk of coronary heart disease," Molecular Biology Reports, vol. 39, no. 2, pp. 1809$1813,2012$.

[88] V. Kolovou, A. Marvaki, A. Karakosta et al., "Association of gender, ABCA1 gene polymorphisms and lipid profile in Greek young nurses," Lipids in Health and Disease, vol. 11, article 62, 2012.

[89] Z. Xiao, J. Wang, W. Chen, P. Wang, and H. Zeng, "Association studies of several cholesterol-related genes (ABCA1, CETP and
LIPC) with serum lipids and risk of Alzheimer's disease," Lipids in Health and Disease, vol. 11, article 163, 2012.

[90] F. Wavrant-De Vrièze, D. Compton, M. Womick et al., "ABCA1 polymorphisms and Alzheimer's disease," Neuroscience Letters, vol. 416, no. 2, pp. 180-183, 2007.

[91] E. B. Neufeld, J. A. Stonik, S. J. Demosky Jr. et al., “The ABCA1 transporter modulates late endocytic trafficking: insights from the correction of the genetic defect in Tangier disease," The Journal of Biological Chemistry, vol. 279, no. 15, pp. 15571-15578, 2004.

[92] E. J. Schaefer, C. B. Blum, R. I. Levy et al., "Metabolism of high density lipoprotein apolipoproteins in Tangier disease," The New England Journal of Medicine, vol. 299, no. 17, pp. 905-910, 1978.

[93] J. Huang, W. Huang, H. Li et al., "Relationship between the I883M polymorphism of ATP-binding cassette transporter 1 gene and cardiovascular disease," Shandong Medical Journal, vol. 9, 2009.

[94] A. Sandhofer, B. Iglseder, S. Kaser, E. Morè, B. Paulweber, and J. R. Patsch, "The influence of two variants in the adenosine triphosphate-binding cassette transporter 1 gene on plasma lipids and carotid atherosclerosis," Metabolism, vol. 57, no. 10, pp. 1398-1404, 2008.

[95] U. Hodoglugil, D. W. Williamson, Y. Huang, and R. W. Mahley, "Common polymorphisms of ATP binding cassette transporter A1, including a functional promoter polymorphism, associated with plasma high density lipoprotein cholesterol levels in Turks," Atherosclerosis, vol. 183, no. 2, pp. 199-212, 2005.

[96] M. K. Jensen, J. K. Pai, K. J. Mukamal, K. Overvad, and E. B. Rimm, "Common genetic variation in the ATP-binding cassette transporter A1, plasma lipids, and risk of coronary heart disease," Atherosclerosis, vol. 195, no. 1, pp. el72-e180, 2007.

[97] I. Porchay-Baldérelli, F. Péan, N. Emery et al., "Relationships between common polymorphisms of adenosine triphosphatebinding cassette transporter Al and high-density lipoprotein cholesterol and coronary heart disease in a population with type 2 diabetes mellitus," Metabolism: Clinical and Experimental, vol. 58, no. 1, pp. 74-79, 2009.

[98] M. E. Brousseau, M. Bodzioch, E. J. Schaefer et al., "Common variants in the gene encoding ATP-binding cassette transporter 1 in men with low HDL cholesterol levels and coronary heart disease," Atherosclerosis, vol. 154, no. 3, pp. 607-611, 2001.

[99] T. Harada, Y. Imai, T. Nojiri et al., "A common Ile 823 Met variant of ATP-binding cassette transporter $\mathrm{Al}$ gene (ABCA1) alters high density lipoprotein cholesterol level in Japanese population," Atherosclerosis, vol. 169, no. 1, pp. 105-112, 2003.

[100] E. J. Schaefer, D. W. Anderson, L. A. Zech et al., "Metabolism of high density lipoprotein subfractions and constituents in Tangier disease following the infusion of high density lipoproteins," Journal of Lipid Research, vol. 22, no. 2, pp. 217-228, 1981.

[101] M. T. Villarreal-Molina, C. A. Aguilar-Salinas, M. RodríguezCruz et al., "The ATP-binding cassette transporter A1 R230C variant affects HDL cholesterol levels and BMI in the Mexican population: association with obesity and obesity-related comorbidities," Diabetes, vol. 56, no. 7, pp. 1881-1887, 2007.

[102] C. A. Aguilar-Salinas, S. Canizales-Quinteros, R. RojasMartínez et al., "The non-synonymous Arg230Cys variant (R230C) of the ATP-binding cassette transporter A1 is associated with low HDL cholesterol concentrations in Mexican adults: a population based nation wide study," Atherosclerosis, vol. 216, no. 1, pp. 146-150, 2011. 
[103] B. Sorrenson, R. J. Suetani, M. J. A. Williams et al., "Functional rescue of mutant ABCA1 proteins by sodium 4-phenylbutyrate," Journal of Lipid Research, vol. 54, no. 1, pp. 55-62, 2013.

[104] S. H. Hong, J. Rhyne, K. Zeller, and M. Miller, "ABCA1Alabama: a novel variant associated with HDL deficiency and premature coronary artery disease," Atherosclerosis, vol. 164, no. 2, pp. 245$250,2002$.

[105] S. Bungert, L. L. Molday, and R. S. Molday, "Membrane topology of the ATP binding cassette transporter ABCR and its relationship to $\mathrm{ABC1}$ and related $\mathrm{ABCA}$ transporters: identification of N-linked glycosylation sites," The Journal of Biological Chemistry, vol. 276, no. 26, pp. 23539-23546, 2001. 


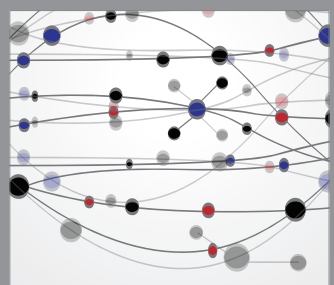

The Scientific World Journal
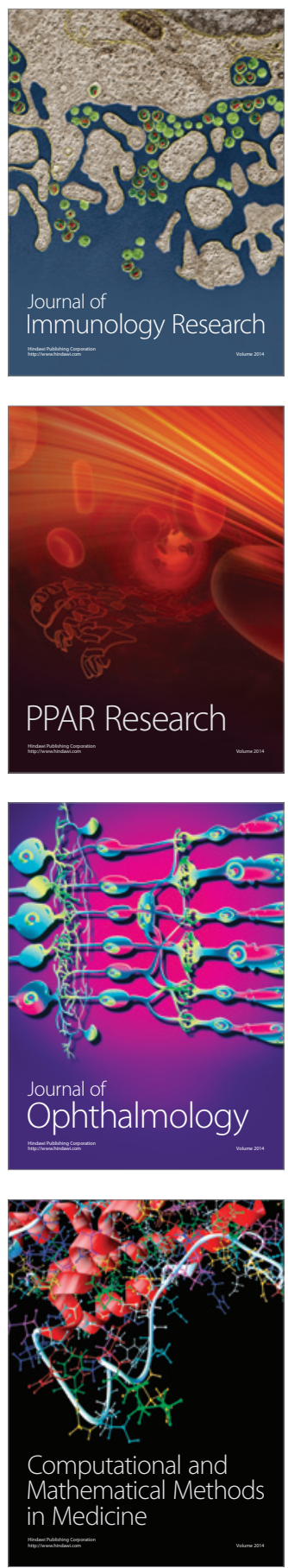

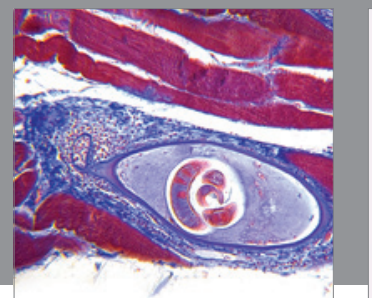

Gastroenterology

Research and Practice
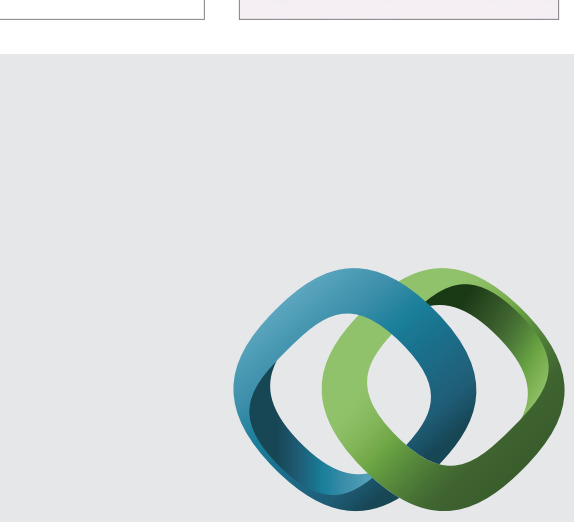

\section{Hindawi}

Submit your manuscripts at

http://www.hindawi.com
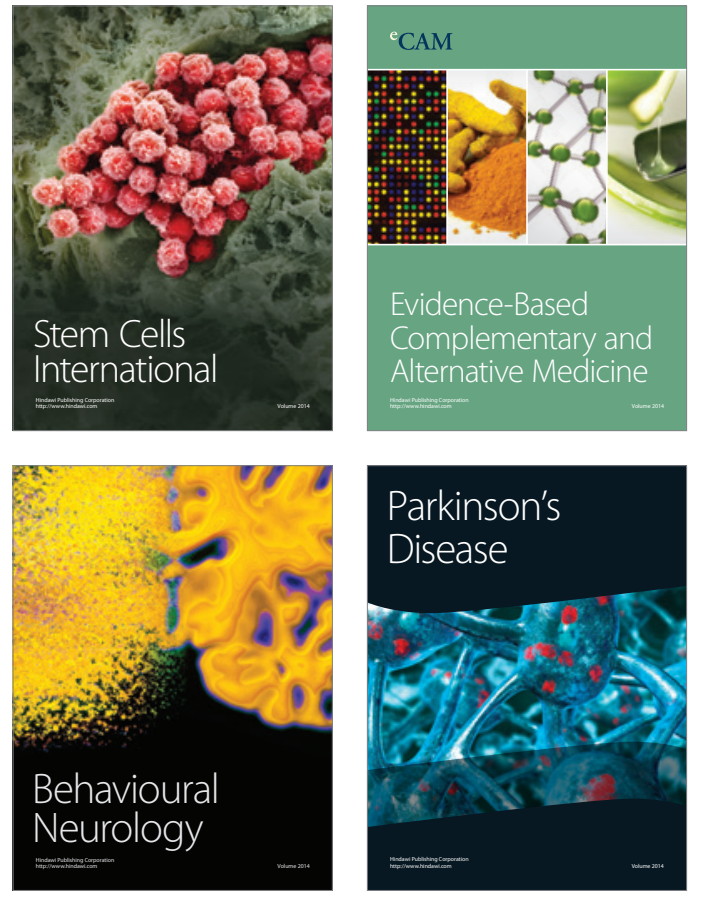
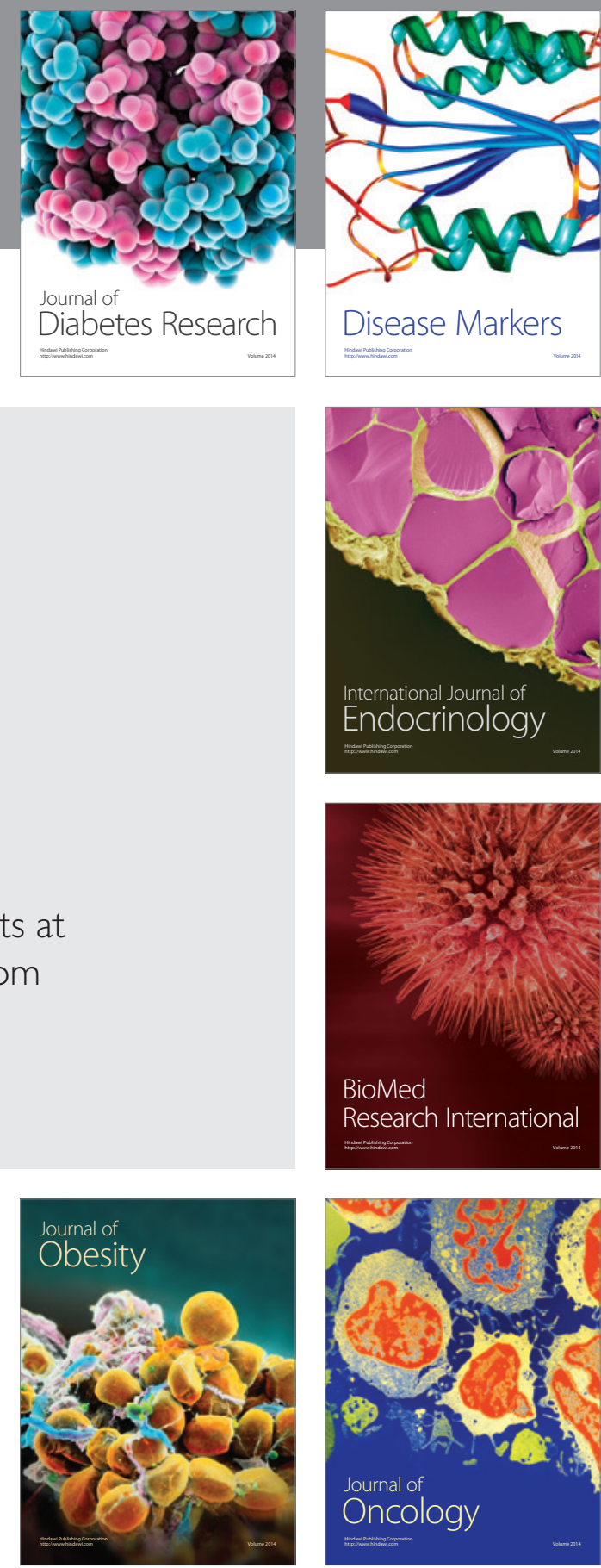

Disease Markers
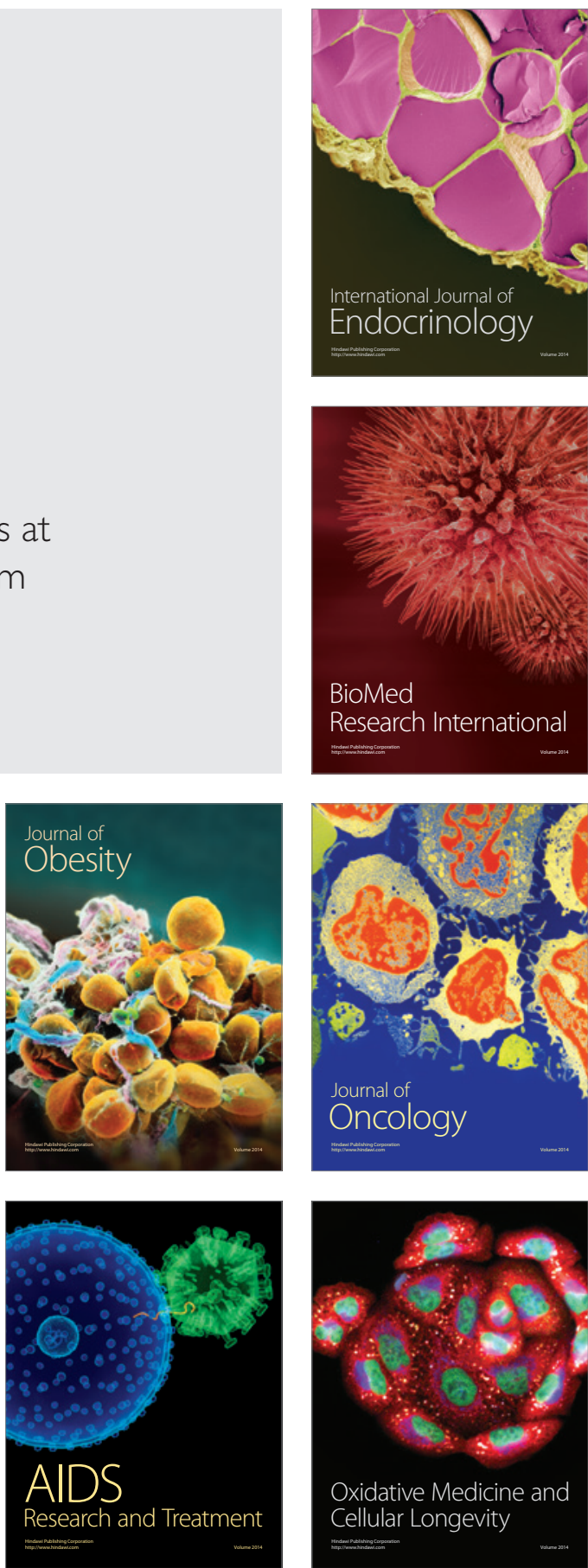